\title{
La estructura de la frase nominal en el mazahua
}

\author{
The structure of the nominal phrase in Mazahua
}

\author{
Armando Mora-Bustos \\ Universidad Autónoma Metropolitana Unidad Iztapalapa, \\ Departamento de Filosofía \\ Gabriela Mora Muñoz \\ Universidad Autónoma Metropolitana Unidad Iztapalapa
}

\begin{abstract}
Resumen
El objetivo de este trabajo es describir la plurales, pronombres, demostrativos, nuestructura de la frase nominal (FN) en mazahua; esta construcción está formada por un núcleo nominal sin modificadores o por un núcleo modificado por prefijos que codifican dimensión y posesión. Los núcleos nominales están modificados por nominales y cláusulas relativas que expresan conceptos de propiedad y cláusulas relativas de diferente naturaleza estructural. Los núcleos nominales coocurren con formas merales y cuantificadores. Este trabajo es eminentemente descriptivo; se enmarca dentro de una metodología funcionalista; los diferentes rasgos de esta estructura están descritos a partir de la correlación de los diferentes niveles de análisis de la lengua. El corpus está integrado por diferente material, esto es, narraciones, historias de vida, cuentos y elicitaciones directas.
\end{abstract}

Palabras clave: sustantivo; frase; modificadores, cuantificadores; cláusulas relativas; otomangue 


\section{Abstract}

The objective of this paper is to describe plural forms, pronouns, demonstratives, the structure of the nominal phrase (NP) numerals, and quantifiers. This work is in Mazahua; this structure is formed by basically descriptive; it is framed within a nominal core with no modifiers, or by a core modified by prefixes that encode for dimension and possession. Nominal cores are modified by nominal and relative clauses that express concepts of property and relative clauses of varying syntactic nature. Nominal cores co-occur with a functionalist methodology; the different features of this structure are described according to correlations across the various levels of language analysis. The corpus is composed of a number of materials, that is, accounts, life stories, stories, and direct elicitations.

Keywords: noun; phrase; modifiers; quantifiers, relative clauses; Otomanguean

Fecha de recepción del artículo: 7 de marzo de 2017 Fecha de recepción de la versión revisada: 5 de diciembre de 2017

Fecha de aceptación: 23 de enero de 2018 


\section{Introducción}

El propósito de este trabajo es describir la estructura de la frase nominal (FN) en el mazahua; específicamente se caracterizarán los núcleos y los modificadores, así como su comportamiento dentro y fuera de la frase nominal. Se describirán las FN integradas por un nominal con y sin modificadores, como en (1). Esta lengua dispone de diferentes recursos para pluralizar el núcleo nominal; por ejemplo, la FN $2 \hat{t}^{\grave{h}} m \dot{t}$, como se observa en (1a), ${ }^{1}$ está modificada con el plural $h i$, mientras que en el núcleo nominal $p^{h} \breve{a} d \hat{t}$, presente en (1b), el sentido de pluralidad lo codifica el artículo jò. De la misma manera, en (1c) el demostrativo plural nùhjó expresa el sentido plural del núcleo nominal báya. Se describirán también los prefijos de dimensión y las marcas de posesión, como en (1d). Los datos del mazahua que se presentan aquí pertenecen a la variante de Crescencio Morales (San Mateo), municipio de Zitácuaro, Michoacán. ${ }^{2}$ Los ejemplos utilizados para ilustrar los diferentes fenómenos gramaticales son parte de narraciones, historias de vida y elicitaciones directas.

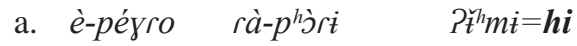
NP-Pedro 3.FUT-cuidar zorrillo=PL
'Pedro cuidará a los zorrillos.' [txt.coyote]
b. $\quad \boldsymbol{j} \grave{o}=p^{h} \check{a} d \grave{t}$
zú-kît'i nà =hníni
$\grave{a}=$ sán.máteo
ART.DEF.PL $=$ caballo
3.PST-entrar ART.INDF.SG $=$ rancho
LOC=San.Mateo
'Los caballos entraron a un rancho de San Mateo.' [txt.nar]
c. пйhjó бáya 'já=Ø-pǒPo=jó
bá? $a=j o ́$
DEM.PL vaca NEG=3.PRS-tener=DEM.PL leche=DEM.PL
'Estas vacas no tienen leche.' [txt.diag]

1 El prefijo $\grave{e}$ - aparece solo con nombres propios.

2 Los datos presentados en este trabajo fueron obtenidos directamente por los autores desde el año 2015 hasta la actualidad; agradecemos la colaboración de Julia Guzmán Reyes y Mercedes Santiago Sámano, hablantes nativas del mazahua de Michoacán. Los ejemplos presentados en el estudio fueron seleccionados de un banco de datos que consiste en narraciones de historia de vida, cuentos de tradición oral, diálogos, entrevistas, elicitaciones directas, etc. A continuación se enumeran los textos que forman parte del corpus: Jò thěze bùzißi 'Tamales de ceniza'; Nu ygəni 'La gallina'; Há yge ßa rí Pàt’akhohme nù sénto 'Cómo hacemos el pulque sende'; Nù mbáfua nù sètiembre 'La fiesta de septiembre', y Jò mìno 'El coyote'. 

d. yú-séyg ${ }^{w} a-t s ' t \quad$ in-ts'i-pápáyande
3.PST-visitar-2.оBJ 1.POS-DIM-abuelo
'Te visitó mi abuelito.' [txt.diag]

Sin pretender, en lo absoluto, realizar una descripción detallada y minuciosa de la estructura de la FN, se presentarán los cuantificadores y los numerales, como los mostrados en (2); en específico, nàpúnk'i 'mucho' y tsínto 'cinco' de (2a), así como la estructura de los compuestos, mbàfímo 'jícara roja' de (2b); los conceptos de propiedad formalizados en cláusulas relativas, como mìp̌ri i 'estaban gordos' de (2c) y las oraciones relativas del tipo k'a nùt'îi màs'ó?o, 'el niño que era malo' de (2d).
a. jò=tsíntfo târehe ø-sì?i nà-púnk'i thố?õ
ART.PL=nueve gallo 3.PRS-comer PRED-mucho maíz
'Nueve gallos comen mucho maíz.' [elcd.bat.cuant]
b. ró-pǒPna nù=mbà-fímo
1.PST-robar ART.DEF.SG=rojo-jícara
'Yo me robé la jícara roja.' [txt.nar]

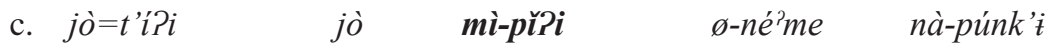
ART.DEF.PL=niño REL.PL PRED.PRS-gordo 3.PRS-bailar PRED-mucho
'Los niños que estaban gordos bailan mucho.' [elcd.bat.cuant]
d. $\boldsymbol{k}^{\prime} \boldsymbol{a}$ nù=t'í?i mà-s'ó?o Ø-pว̌rna nù=t'ěne
REL.SG ART.DEF.SG-niño PRED.PST-malo 3.PST-robar ART.DEF.SG=juguete
'El niño que era malo se robó el juguete.' [elcd.bat.rel]

La importancia de este trabajo radica en que poco o casi nada se sabe sobre este tema en la lengua mazahua contemporánea; si bien dentro del subgrupo otopame se encuentran los trabajos de Palancar (2009) sobre el otomí de San Ildefonso Tultepec, Querétaro, y el de Gibson y Bartholomew (1979) acerca del pame central. En términos muy generales, la frase nominal ha sido descrita en diferentes variedades del mazahua por Stewart (1966), Amador Hernández (1976), López Marín (2002), Knapp (2013) y Vargas Bernal (2013). 


\section{Aspectos generales de la lengua}

El mazahua es una lengua otomangue y, junto con el otomí, matlatzinca, tlahuica, pame y chinanteco, constituye el subgrupo otopame. Se trata de una lengua tonal; los tonos de nivel que se anclan en los prefijos verbales permiten identificar a la persona gramatical y el tiempo, el aspecto y el modo; igualmente, los tonos de nivel que se anclan en los prefijos de posesión permiten identificar al poseedor. Es una lengua nominativo-acusativa y de marcación en el núcleo, es decir que las relaciones gramaticales se codifican en el núcleo verbal mediante un conjunto complejo de afijos. Los prefijos codifican tiempo, aspecto y modo y hacen correferencia con la frase nominal que expresa al sujeto gramatical; los sufijos exhiben las relaciones gramaticales objeto y objeto indirecto. El orden básico de constituyentes en oraciones transitivas corresponde a vos, como en (3a); en construcciones con verbos ditransitivos a svo-OI, como en (3b), y en oraciones intransitivas a SV(X), como en (3c). Estas disposiciones de la distribución del orden de constituyentes varían por condicionamientos discursivos.

$\mathbf{V}$

$\mathbf{0}$

$\mathbf{S}$

a. $\quad \gamma u$-jòd

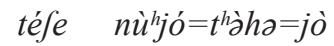

$j o \grave{o}=\grave{i n}-t^{\prime} \supset \int-p^{h} a d \grave{t}$

3.PST-pisar todo DEM.PL=surco=DEM.PL ART.DEF.PL=2.POS-blanco-caballo

'Tus caballos blancos pisaron todos esos surcos.' [txt.nar]

$\mathbf{s}$

b. in-k $k^{w} \check{r} r m a$ 1.Pos-hermano o nà-púnk’’ mério

dinero
OI

$$
j \grave{o}=t i ? i
$$

ART.DEF.PL $=$ niño

'Mi hermano les robará mucho dinero a los niños.' [elcd.ord]
$\mathbf{S}$
$\mathbf{v}$
$\mathbf{x}$
c. $n \grave{u}=h_{m} \tilde{y} \tilde{\tilde{o}}$
$n \grave{u}=6 \grave{z} z O$
$\varnothing-k^{w} a ́ n a$
t'ike $k^{\prime} \grave{a}=t \grave{j} j \varepsilon$
ART.DEF.SG=coyote
ART.DEF.SG=hombre
3.PRS-correr poco
LOC=bosque
'El coyote del hombre corre poco en el bosque.' [txt.elcoyote]

Para fines de este artículo se han retomado los siguientes trabajos: la estructura de la FN se fundamenta en Givón (2001), Rijkhoff (2002), Plank, Bossong y Comrie (2003), Kroeger (2005) y Dryer (2013); el tratamiento descriptivo de los conceptos de propiedad se ha realizado a partir de Smith-Stark (2004) y Pa- 
lancar (2006); la configuración de las cláusulas de relativo se ha implementado con lo desarrollado por Keenan (1985) y Andrews (2007); entre otros temas, se ha realizado el tratamiento del concepto de posesión con los planteamientos de Seiler (1983), Aikhenvald y Dixon (2013). Todos estos trabajos, a excepción de Rijkhoff (2002), se inscriben bajo la perspectiva funcional-tipológica.

Este artículo está organizado de la siguiente manera: 33 nominales sin modificador, $\$ 4$ prefijos de dimensión, $\S 5$ formas plurales, $\S 6$ artículos, $\$ 7$ demostrativos, $\S 8$ numerales, $\$ 9$ cuantificadores, $\$ 10$ conceptos de propiedad, $\$ 11$ compuestos, $\$ 12$ cláusulas relativas, $\$ 13$ posesión nominal y $§ 14$ frase posesiva compleja.

\section{Nominales sin modificador}

En la lengua mazahua aparecen FN formadas por un nominal sin modificador, como en (4) y (5). Estas FN no presentan ningún tipo de restricción semántica; pueden aparecer nominales contables, como en (4), 2jó?Po 'perro', ndǒra 'duraznos', rêk ${ }^{w} a$ 'burro', bèzo 'hombre', y no contables, como en (5), tóp’’ 'dinero', náfôma 'arena', sǐßi 'fuego', záphadì 'zacate'.

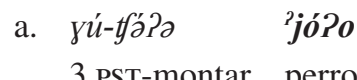

'Montó al perro.' [elcd.ord]

b. nù=ndòpàrì $\quad \varnothing$-sà?a ndŏra

ART.DEF.SG=zopilote 3.PRS-comer durazno

'El zopilote se come los duraznos.' [txt.nar]

c. ø-Pǐ̌h $\quad$ rêk $\boldsymbol{k}^{w} \boldsymbol{a}$ nà-púnki

3.PRS-dormir burro PRED-mucho

'Los burros duermen mucho.' [elicd.cuant]

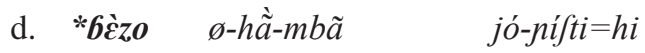

hombre 3.PRS-mirar-3.DAT ART.DEF.3.POS.PL-CABELLO=PL

'El hombre le mira su cabello.' [elcd.ord]

a. $\quad u^{u}-m b \check{s}=h i \quad$ tóp'i

3.PST-robar=PL dinero

'Se robaron el dinero.' [txt.diag] 


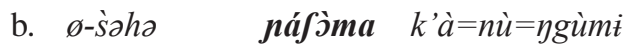
3.PRS-llegar arena LOC $=$ ART.DEF.SG $=$ casa
'Llegó la arena a la casa.' [txt.diag]

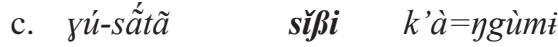
3.PST-quemar fuego $\mathrm{LOC}=$ casa
'El fuego quemó la casa.' [txt.diag]
d. “záph adi ró-pènt'i mí-ndzăra
zacate 1.PST-agarrar TADV-ayer
'Yo agarré el zacate ayer.' [elcd.ord]

La posición de las FN formadas por nominales sin modificador, como en (4) y (5), es la postverbal; no pueden aparecer en la preverbal, como en (4d) y (5d). Téngase en cuenta que si bien, en la lengua, el orden de constituyente (vos, SVO-OI, SV(X) puede variar por motivaciones pragmáticas, el espectro de movimiento de las FN sin modificador se restringe al postverbal.

\section{Prefijos de dimensión en las FN}

Los morfemas ligados $t s^{\prime} i ́$, tà y $t s^{\prime} o ́$ aparecen prefijados al nominal; estos exhiben un sentido de dimensión, esto es, diminutivo en (6), aumentativo en (7) y tamaño medio en (8); estos prefijos se ubican en la posición más interna con respecto al sustantivo nuclear que modifican.

(6) Diminutivos
a. rá-hàbə

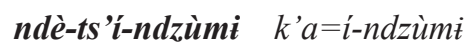
1.FUT-construir
PART-DIM-casa
LOC $=1$. POS-casa
'Le voy a construir un techito a mi casa.' [elicd.dime]
b. in-ts'í-mbàre yú-kóPbí è-hòsé
1.POS-DIM-cántaro 3.PST-tapar NP-Jose
'Mi cantarito lo tapó José.' [elicd.dime]
c. nù=ts'í-ygổni
ø-sà?a in-ţhớ?
ART.DEF.SG=DIM-pollo 3.PRS-comer 1.POS-maíz
'El polluelo se come mi maíz.' [elicd.dime]




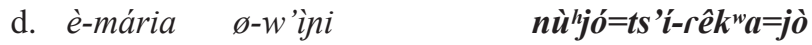
NP-María 3.PST-dar.de.comer DEM.PL=DIM-burro=DEM.PL
'María le dio de comer a esos burritos.' [elicd.ord]

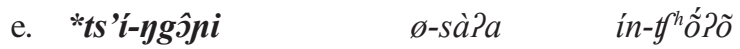
ART.DEF.SG=DIM-pollo 3.PRS-comer 1.POS-maíz
'El polluelo se come mi maíz.' [elicd.dime]

En una posición externa, con respecto a los prefijos de dimensión, se ubican los afijos de posesión, como en (6b), o el partitivo, como en (6a). En la posición más externa al núcleo aparecen proclitizados los artículos, como en (6c), y los demostrativos, como en (6d). El constituyente oracional expresado en una FN integrada por un núcleo que está modificado por un prefijo de dimensión más uno que exprese posesión o un artículo o un demostrativo se puede mover dentro del espectro de la oración; es decir, las frases nominales que codifiquen una relación gramatical (sujeto, objeto y objeto indirecto) aparecen en cualquier posición dentro de la oración.

(7) Aumentativos

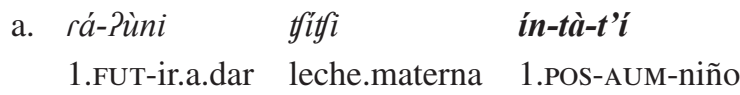

'Le voy a dar leche a mi hijo mayor.' [txt.nar]

b. ìn-tà-mèsa ró-pǒru

2.POS-AUM-mesa 1.PST-cambiar

'Cambié tu mesota.' [elicd.dime]

c. $\boldsymbol{n u}=t \grave{u}-t^{h} \grave{u} \mathbf{u} \tilde{\boldsymbol{t}}$ rà- ${ }^{h}$ wánt'ti

ART.DEF.SG=AUM-sombrero 3.FUT-escoger

'Escogerá el sombrerote.' [elicd.dime]

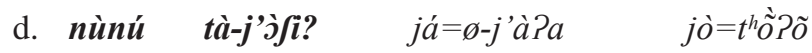

DEM.SG AUM-gusano PTL=3.PST-podrir $\quad$ ART.DEF.PL $=$ maíz

'Este gusanote ya pudrió los maíces.' [txt.nar]

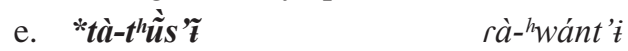

ART.DEF.SG=AUM-sombrero 3.FUT-escoger

'Escogerá el sombrerote.' [elcd.tam] 
Los ejemplos (6e), (7e) y (8e) son agramaticales porque las FN no pueden aparecer modificadas solo por un prefijo de tamaño, sino que debe coaparecer un morfema de posesión, un artículo o un demostrativo.

(8) Tamaño medio

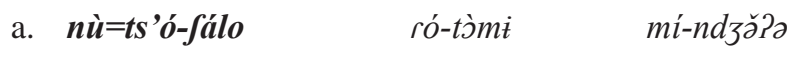

ART.DEF.SG=MED-jarro 1.PST-comprar TADV-ayer

'El jarro mediano lo compré ayer.' [txt.nar]

b. in-ts'ó-t'í?i yú-?ě̃hẽ à=hníni

1.POS-MED-niño 3.PST-venir LOC=pueblo

'Mi hijo mediano vino al pueblo.' [txt.nar]

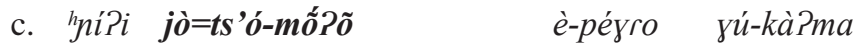

tres ART.DEF.PL=MED-pescado NP-Pedro 3.PST-pescar

'Pedro pescó tres pescados medianos.' [txt.nar]

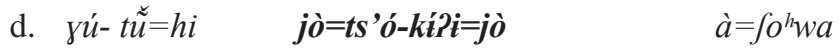

3.PST-traer=PL DEM.PL=MED-frijol=DEM.PL LOC=Zitácuaro

'Trajeron estos frijoles medianos de Zitácuaro.' [txt.nar]

e. *ts'ó-mố? $\tilde{\boldsymbol{o}}$ è-péyro yú-kà?ma

ART.DEF.PL=MED-pescado NP-Pedro 3.PST-pescar

'Pedro pescó pescados medianos.' [txt.nar]

\section{Plurales}

La lengua mazahua dispone de diversos recursos morfosintácticos para expresar la pluralidad, esto es, enclíticos de número, artículos y demostrativos plurales y cuantificadores. En este trabajo se describirán por separado cada una de estas unidades gramaticales. Los morfemas que codifican plural se presentan en el Cuadro 1.

Cuadro 1. Enclíticos de número

\begin{tabular}{ccccc}
\hline Persona & Plural & Dual & Dual Exclusivo & Plural Exclusivo \\
\hline 1 & $=h i$ & $=\beta i$ & $=6 \mathrm{e}$ & $={ }^{\mathrm{h}} \mathrm{me}$ \\
2 & $=\mathrm{hi}$ & $=\beta \mathrm{i}$ & & \\
3 & $=\mathrm{hi}$ & $=\beta \mathrm{i}$ & & \\
\hline
\end{tabular}


Estos enclíticos que codifican plural y dual aparecen en (9). Los nominales modificados por el enclítico de plural, generalmente, no están modificados por un artículo o por un demostrativo, como mápehi 'morrales' en (9a), y 'mû́bi 'dos chilacayotes' en (9c). Con el propósito de que la FN se ubique en posición preverbal, como en (9d), el núcleo de la FN que expresa el plural debe estar modificado por un prefijo de posesión o por un artículo demostrativo; esta estructura es similar a la que aparece en posición posverbal, como en (9b); de lo contrario se genera una construcción agramatical, como en $n d \hat{\varepsilon} n t y^{h}{ }^{\prime} b e$ de $(9 \mathrm{e}){ }^{3}$
a. è-fúßa ø-tôt'í mápe $=\boldsymbol{h i}$
NP-Juan 3.PRS-encontrar morral=PL
'Juan encuentra los morrales.' [txt.diag]
b. $n \grave{u}=6 \check{c} n a$ rà-på̀r $\tilde{a}$
ín-ty'éhe ${ }^{h}$ me
ART.DEF.SG=señora 3.FUT-conocer 1.POS-cerro=PL.EXCL
'La señora conocerá nuestros cerros.' [txt.nar]
c. $n \grave{u}=s^{\prime} a n t^{h} \varepsilon$
rà-?ókì
${ }^{h} \boldsymbol{m} \hat{\hat{u}}=\boldsymbol{\beta i}$
ART.DEF.SG $=$ brujo 3.FUT-cortar chilacayote $=$ DL
'El brujo cortará dos chilacayotes.' [elic.lxc]
d. $\quad \boldsymbol{i}$-ndzênthi $i=6 e$ $\varnothing-t s^{h} \grave{t} n t ' \dot{t}$ $k^{\prime} a=n \grave{u}=m c^{\prime} h \varepsilon$
1.POS-borrego=DL.EXCL 3.PST-ahogar $\quad$ LOC=ART.DEF.SG-pozo
'El borrego de nosotros dos se ahogó en el pozo.' [txt.nar]

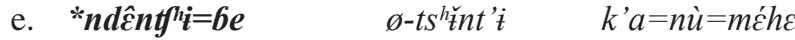
PL.borrego=DL.EXCL 3.PST - ahogar LOC=ART.DEF.SG-pozo
'El borrego de nosotros dos se ahogó en el pozo.' [txt.nar]

Es importante resaltar que si bien los enclíticos ilustrados en el Cuadro 1 codifican plural; estos pueden o no coocurrir con los artículos que codifican plural, como en (10). El plural se expresa con el enclítico de número hi en (10a), o con un artículo plural jò en (10b); estos pueden coaparecer en la misma FN, jò ...hi en (10c), o en la oración, jò...hi en (10d); hi en (10d) hace referencia con la frase

3 En este trabajo no se describen los plurales de los conceptos colectivos; véase al respecto Knapp (2008). 
que expresa el sujeto, mientras que en (10e) el enclítico hi, que igualmente está indexado en el verbo, hace referencia con la frase que expresa al objeto directo.

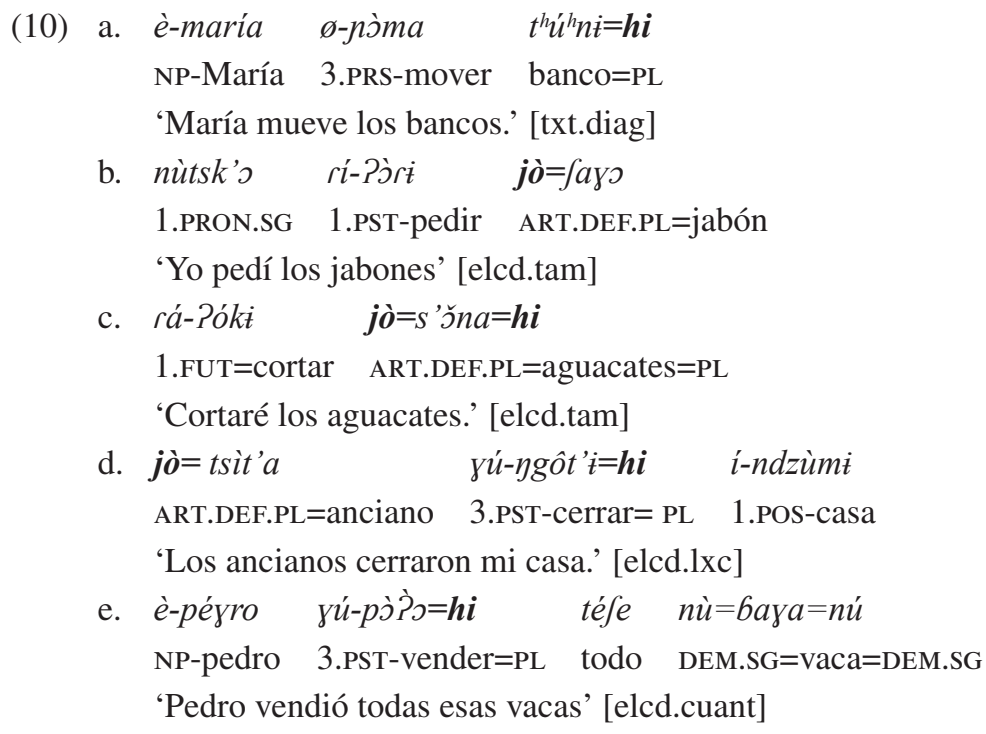

En las FN modificadas con demostrativos plurales, nùhjój'óPojò 'estos perros' de

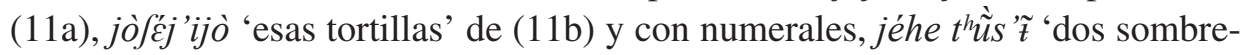
ros' en (12a), tŷtวì Đgว̂ni 'ocho pollos' en (12b), no coaparece la marca de número plural $h i$; la ocurrencia de $h i$ genera construcciones agramaticales, como

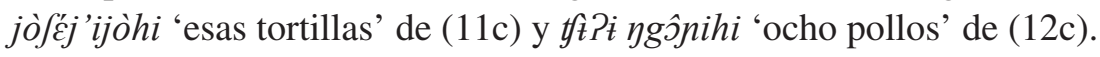
a. nù $h_{j o ́} \quad j^{\prime} \boldsymbol{o ́} P o=j \grave{o}$

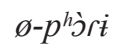
in-tนô??
DEM.PL perro= DEM.PL 3.PRS-cuidar 1.POS-maíz
'Estos perros cuidan mi milpa.' [txt.diag]
b. nù=ndèj'e
yú-sã́t'ã

$$
\boldsymbol{j} \grave{\boldsymbol{o}}=\int \hat{c}^{\prime} \boldsymbol{j} \boldsymbol{i}=\mathbf{j} \grave{\boldsymbol{o}}
$$
ART.DEF.SG=comal 3.PST-quemar DEM.PL-tortilla=DEM.PL
'El comal quemó esas tortillas.' [txt.las tortillas]
c. *nù=ndèj'e
yú-sát' 'ã
$j \grave{o}=\int_{\varepsilon}^{\prime} j{ }^{\prime} i=j \grave{o}=h i$
ART.DEF.SG=comal 3.PST-quemar DEM.PL-tortilla=DEM.PL $=$ PL
'El comal quemó esas tortillas.' [txt.las tortillas] 
(12)
a. nù=ndà̃ $m \tilde{a} \quad$ yú-pà?a jéhe thà̀us $\tilde{t}$
ART.DEF.SG=viento 3.PST-aventar dos sombrero
'El viento aventó dos sombreros.' [txt.diag]

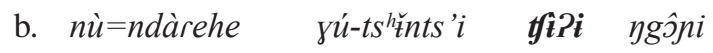
ART.DEF.SG=río 3.PST-ahogar ocho pollo
'En el río se ahogaron ocho pollos.' [txt.diag]
c. *nù=ndàrehe yú-tsȟ̆nts'i tyîi $\boldsymbol{y g} \hat{\boldsymbol{g}} \boldsymbol{n} \boldsymbol{n} \boldsymbol{i}=\boldsymbol{h i}$
ART.DEF.SG=río 3.PST-ahogar ocho pollo=PL
'En el río se ahogaron ocho pollos.' [txt.diag]

Obsérvese que el enclítico plural $h i$ aparece indexado tanto en el verbo como en el nominal. La coocurrencia de esta marca de plural es jerárquica, es decir, si el sujeto gramatical expresa pluralidad, $h i$ se enclitiza en el verbo, como en (12d); por su parte, si el objeto denota una entidad plural, $h i$ se enclitiza en el nominal nuclear, como en (12e). En los contextos en donde el sujeto y el objeto son entidades plurales, como en (12f), $h i$ se enclitiza en el verbo y hace correferencia con la frase nominal que expresa al sujeto. Finalmente, si la frase nominal sujeto expresa un sentido singular y la frase objeto expresa plural, $h i$ se enclitiza en el verbo, como en $(12 \mathrm{~g})$, y hace correferencia con el objeto directo.
d. jò=tsit'a

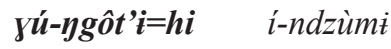
ART.DEF.PL $=$ anciano 3. PST-cerrar $=\mathrm{PL} \quad 1$. POS-casa
'Los ancianos cerraron mi casa. [elcd.lxc]
e. è-màría ø-n’̀ma $\boldsymbol{t}^{h} \boldsymbol{u}^{h} \boldsymbol{n} \boldsymbol{i}=\boldsymbol{h i}$
NP-María 3.PRS-mover banco=PL
'María mueve los bancos.' [txt.diag]
f. rá-Póki $=\boldsymbol{h i} \quad j o ̀=s ’ ว ̌ n a$
1.FUT $=$ cortar $=$ pl ART.DEF.PL=aguacates
'Cortaremos los aguacates.' [elcd.lxc]

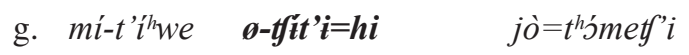
1.POS-hija 3.PRS-cocinar=PL ART.DEF.PL=pan
'Mi hija cocina los panes' [elcd.lxc]




\section{Artículos}

A lo largo de este trabajo no se usa la etiqueta de artículo en función del morfema que codifican estrictamente definitud y especificidad, sino como los morfemas proclíticos, que coocurren libremente con demostrativos y con morfemas que codifican dimensión, posesión, y cuantificación (Dryer, 2007). En este orden de ideas, los demostrativos expresan una función deíctica y coocurren en los mismos contextos de los artículos.

Presentamos en el Cuadro 2 el repertorio de artículos definidos e indefinidos en su forma singular y plural.

\begin{tabular}{|c|c|c|}
\hline & SINGULAR & PLURAL \\
\hline Definido & $\begin{array}{l}\text { nù= } \\
\text { [nù= =nù] }\end{array}$ & $\begin{array}{l}\mathrm{jò}= \\
{[\mathrm{joj}=\quad=\mathrm{jo}]}\end{array}$ \\
\hline Indefinido & $\begin{array}{l}\text { nà = } \\
\text { [nà= =nà] }\end{array}$ & nù'jó= \\
\hline
\end{tabular}

Los artículos son formas libres que se proclitizan en el nominal modificado. ${ }^{4} \mathrm{De}$ (13) a (15) se presentan ejemplos en los que se ilustra la posición de estas unidades dentro de la FN, esto es: nù’jóPo 'el perro' en (13a), jòfé̉ji 'las tortillas' en (14a), nàndènfì 'una cebolla' en (15b).

4 A continuación se enlistan los nominales que pueden aparecer sin artículo o, por el contrario, aparecen con el artículo singular o plural:

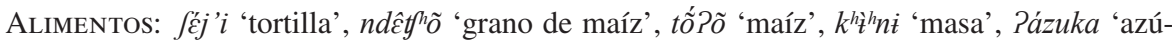
car', káphe 'café', Pố?õ 'sal', hnôna 'comida', ndóra 'durazno', lúlu 'fruta', Páro 'arroz', th'mety' $i$ 'pan', ygére 'carne'.

Líquidos: $\quad t^{\prime}$ 'ose $\tilde{e}^{h} m \tilde{e}$ 'pulque', $t$ 'è $e^{h} m e$ 'atole', $6 a$ ?a 'leche', $t$ 'âp ${ }^{h} \dot{t}$ 'alcohol', Páset'e 'aceite', méska 'mezcal'.

Materiales: ndzáPa 'leña', náfôma 'arena', nòna 'cal', mbèhomì 'lodo', théme 'carbón', Pádoße 'adobe', s 'áphari 'zacate'.

Otros: $\quad$ bítu 'ropa', kănaßi 'basura', tére 'gente'. 
De igual forma, los artículos tienen la propiedad de ubicarse, dentro de la misma FN, como proclíticos y como cierres oracionales, ${ }^{5}$ nùbżzo...nù 'el hombre' en (13b), jòpã́h hã ...jò 'las camisas' en (14b) y nàndènfí... nà 'una cebolla' en (15b). La duplicación del artículo se genera en las oraciones en donde la FN se encuentra en posición preverbal. El artículo definido plural y el artículo indefinido singular se enclitizan en la última unidad gramatical de la oración, ya sea una relación gramatical, un adjunto o un verbo. Los artículos de cierre oracional son opcionales y por sí solos no tienen una función específica dentro de la FN. La agramaticalidad de la construcción de (13c) está asociada con el hecho de que el cierre oracional ocurre solo en el orden svo y no en vos.

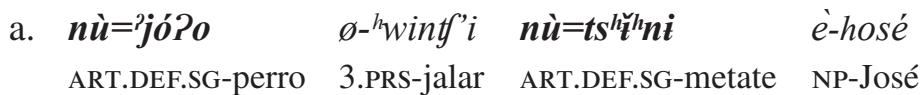

'El perro jala el metate de José.' [elcd.ord]

b. nù= $6 \grave{\varepsilon} z o$ yú-fàt-kì in- $k^{h w} a ́$ Pa $=$ nù

ART.DEF.SG=hombre 3.PST-bañar-1.DAT 1.POS-conejo=ART.DEF.SG

'El hombre bañó a mi conejo.' [elcd.lxc]

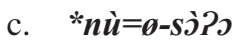

$n \grave{u}=t^{\prime} e^{h} m e$

$n \grave{u}=t \grave{\imath} \mathrm{P} i=n \grave{u}$

ART.DEF.SG=3.PST-beber ART.DEF.SG=atole

ART.DEF.SG=niño=ART.DEF.SG

'El niño bebe atole.' [elcd.lxc]

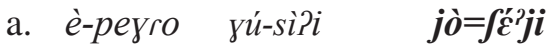
NP-pedro 3.PST-comer ART.DEF.PL-tortilla
'Pedro se comió las tortillas' [txt.las tortillas]
b. $\quad \boldsymbol{j} \boldsymbol{o}=\boldsymbol{p} \tilde{\boldsymbol{a}}^{h} \boldsymbol{n} \tilde{\boldsymbol{a}}$
ø-放 $\tilde{\imath}=\mathbf{j} \boldsymbol{j}$
ART.DEF.PL=camisa 3.PRS-apestar=ART.DEF.PL
'Las camisas apestan.' [txt.diag]

5 En este trabajo cierre oracional es entendido en términos morfosintácticos. Un conjunto de enclíticos aparecen indexados en la última palabra que se encuentra en el extremo derecho de una oración. La oración simple es entendida como aquella unidad sintáctica que está formada por un verbo y las relaciones gramaticales que este requiera, esto es, junto al verbo una oración puede configurarse como la unidad gramatical que expone un sujeto, un objeto directo, un objeto indirecto y una serie de adjuntos de carácter opcional u obligatorio. 
(15)
a. aygèze yú-sà?a nà=p'èndzehe
3.PRON.SG 3.PST-comer ART.INDF.SG-guayaba
'Ella se comió una guayaba.' [elcd.lxc]

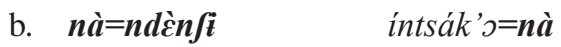

ART.INDF.SG=cebolla 1. PRON.POS.SG=ART.INDF.SG

'Una cebolla es mía.' [elcd.lxc]

Esta duplicación se genera con el artículo definido plural y con el artículo indefinido singular, como en (13b), (14b) y (15b); el artículo indefinido plural $n \grave{u} h j o ́$ bèzo en (16a) no puede aparecer en cierre de oración, como en (16b). El

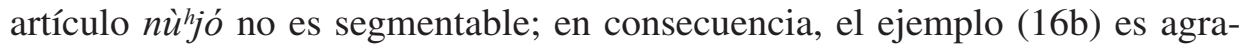
matical; por otra parte, no es posible en (16c) considerar la forma reducida jó como enclítico, demostrativo plural, que se indexa como cierre oracional en minánajó 'tu mamá'.
a. $\boldsymbol{n u ̀ h o ́ ~}$

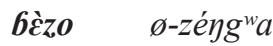
mì-nána
ART.INDF.PL hombre 3.PRS-saludar 2.POS-mamá
'Unos hombres saludan a tu mamá.' [txt.diag]
b. *nùujó
бѐzo ø-zéyg ${ }^{w} a$
mì-nána $=$ nù hjó
ART.INDF.PL hombre 3.PRS-saludar 2.POS-mamá=ART.INDF.PL

'Unos hombres saludan a tu mamá.' [txt.diag]
c. *nù hjó
bèzo ø-zéng ${ }^{w} a$
mì-nána $=$ jó
ART.INDF.PL hombre 3.PRS-saludar 2.POS-mamá=DEM.PL

'Unos hombres saludan a tu mamá.' (Lit. 'Esos hombres saludan a tu mamá.') [txt.diag]

Junto a los artículos ejemplificados en el Cuadro 2, la lengua dispone de dos artículos adicionales codificados en morfemas libres. Los artículos k'òjò y k'ànù codifican un sentido plural, como en (17a), y uno singular, como en (17b).
a. $\quad j a ́=r a ́-h j a ́ s i$
k'òjò
séntfo
PTL=1.FUT-cocer ART.DEF.PL pulque sende
'Vamos a cocer los pulques sendes' [txt.el pulque sende] 

b. ró-hósmì =’ja k'ànù bòzißi
1.PST-cocer=PTL ART.DEF.SG ceniza
'Ya cocimos la ceniza' [txt.los tamales de ceniza]

\section{Demostrativos}

Los demostrativos singulares y plurales se dividen en dos grupos; en el primero aparecen las formas libres y en el segundo las formas ligadas o reducidas. Las formas libres son proclíticos que morfológicamente están compuestos por dos morfemas. El conjunto de demostrativos en su forma singular y plural está resumido en el Cuadro 3.

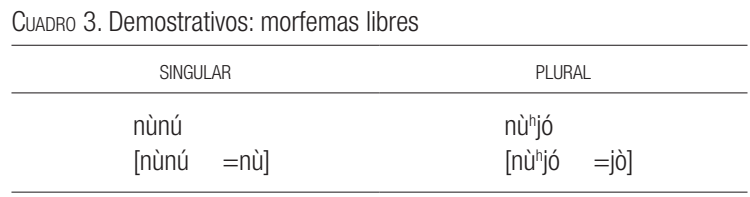

El demostrativo singular nùnú está formado por la iteración del artículo definido singular, como en (18); mientras que el demostrativo plural nùhjó, de (20), está formado por un artículo singular y un artículo plural. La forma léxica del demostrativo nùnú se proclitiza al nominal, nùnú ndènda 'esas tiendas' en (18a); opcionalmente, la forma reducida de este demostrativo, nù, se enclitiza en el nominal, nùnú rek'anù 'esos burros' en (18b). Téngase en cuenta que hay un cambio en el tono de la forma reducida del demostrativo nú; esto debido a que se copia el tono de la última sílaba del demostrativo libre nùnú ygùminú 'esta casa' en (18c).
a. รú-mb̌̌ว nùnú ndènda
3.PST-vender DEM.SG tienda
'Se vendió esa tienda.' [elcd.ord]

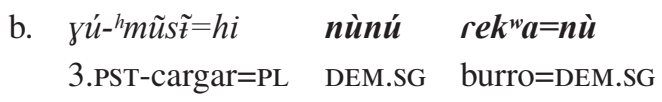
'Cargaron a ese burro' [txt.diag] 


\section{c. è-рѐуго уú-ndét'ti nùnú घgùmi $=$ nú \\ NP-pedro 3.PST-quemar DEM.SG casa=DEM.SG \\ 'Pedro quemó esta casa.' [txt.diag]}

Por otra parte, el demostrativo nùnú expresa una relación gramatical, objeto directo, como en (19a), y sujeto, como en (19b).
a. nùnú è-fúßa rà-mbé=nú
DEM.SG NP-Juan 3.FUT-usar=DEM.SG
'Juan usará esto.' [txt.diag]
b. nùnú rà-mbé $=\gamma \supset \quad \grave{e}-\int u ́ \beta a$
DEM.SG 3.FUT-usar=1.ENF.SBJ NP-Juan
'Esta la usará Juan.' [txt.diag]

El comportamiento gramatical de la forma léxica del demostrativo plural nù jó es similar a la mostrada por el demostrativo singular nùnú. La forma léxica del demostrativo plural aparece en posición prenominal, como en (20a); mientras que la forma reducida, jó, se enclitiza en el nominal, como en (20b). En ambos demostrativos, singular y plural, la forma reducida tiene un carácter enfático.
a. nùhjó nobîini ø-pèzzi
nù=ndéhe
DEM.PL buey
3.PRS-tener.sed
ART.DEF.SG=agua

'Estos bueyes tienen sed.' [txt.lxc]
b. $n \grave{u}=6 \check{n} n a$ rà-fós' $i$
nùhjó Garika=jó
ART.DEF.SG=señora 3.FUT-destapar DEM.PL barril=DEM.PL

'La señora destapará estos barriles.' [elcd.tam]

La forma léxica del demostrativo plural $n u_{\text {hjó }}$, de (21), también expresa una relación gramatical; generalmente, la posición de estos demostrativos es la preverbal, esto si el demostrativo aparece en función de objeto, como en (21a), o sujeto, como en (21b).
a. nù hjó ró-t’̀mí mì-ndză?a
DEM.PL 1.PST-comprar TADV-ayer
'Estas las compré ayer.' [txt.diag] 


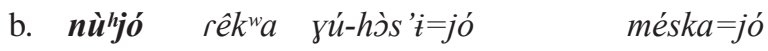

DEM.PL burro 3.PST-lamer=DEM.PL mezcal=DEM.PL

'Esos burros lamieron el mezcal.' [elid.lxc]

El repertorio de las formas reducidas de los demostrativos es muy amplio, véase el Cuadro 4.

CUADRO 4. Demostrativos: formas reducidas

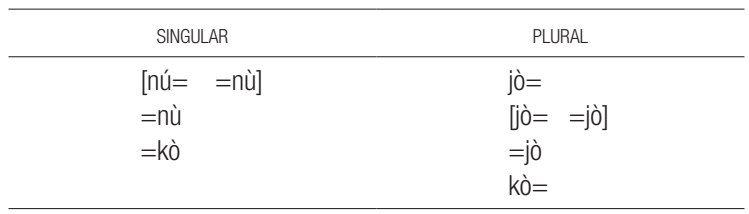

El demostrativo núnù se puede dividir en dos segmentos, como nù ${ }^{h} w \tilde{a}^{h} m a \tilde{n} u ́$ 'esta milpa' en (22a). El primer segmento aparece como proclítico y el segundo como enclítico del nominal; en (22b), este demostrativo se proclitiza en el nominal y la forma reducida nù se enclitiza en el nominal; el enclítico nù se duplica en el núcleo verbal de la construcción y en el constituyente que se encuentra en la parte final de la oración; en esta última posición, aparece como cierre oracional.

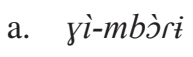

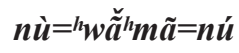
2.PRS-cuidar
DEM.SG-milpa=DEM.SG
'Cuida esta milpa.' [txt.diag]

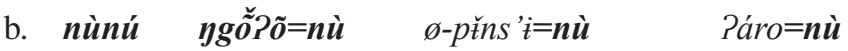
DEM.SG ratón=DEM.SG 3.PRS-oler=DEM.SG arroZ=DEM.SG
'Este ratón huele el arroz.' [elcd.lxc]

La forma reducida de este pronombre aparece como cierre oracional, rófáfinù 'esa rosa' en (23a) y kaníst'anù 'esta canasta' en (23b); esto ocurre en los contextos en donde el nominal al que modifica se encuentra en posición final de oración.
a. mí-nána
Ø-p̌ns $\dot{t}$
rófáfi=nù
1.POS-mamá 3.PRS-oler rosa=DEM.SG
'Mi mamá huele esa rosa.' [elcd.ord] 
b. ró-ygwáre kaníst'a=nù

1.PST-tirar canasta=DEM.SG

'Tiré esta canasta.' [elcd.tam]

El demostrativo plural jò se enclitiza en el nominal al que modifica, como en (24); la FN a la que modifica se mueve libremente dentro de la oración, esto es, la FN ocurre en posición preverbal, como en (24a), o en la posverbal, como en (24b).

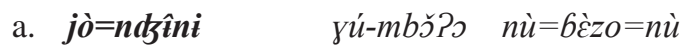

DEM.PL=buey3.P ST-vender $\quad$ DEM.SG=hombre $=$ DEM.SG

'Estos bueyes los vendió ese señor.' [txt.diag]

b. yú-sìi $\boldsymbol{j} \boldsymbol{j} \boldsymbol{o}=\boldsymbol{y} \boldsymbol{g} \grave{\boldsymbol{m}} \boldsymbol{i}$

3.PST-comer DEM.PL $=$ calabaza

'Se comieron estas calabazas.' [txt.diag]

Asimismo, este demostrativo plural se proclitiza y se enclitiza en el nominal, como jòndifujò 'esas señoras' en (25a); igualmente aparece como cierre oracional siempre que el nominal al que hace referencia se encuentre en posición preverbal y al mismo tiempo esté modificado por este demostrativo, como en (25b).
a. $n \grave{u}=s^{\prime} \grave{\partial} 6 \partial=n \grave{u}$
jú-h ${ }^{h}$ wáti
$\boldsymbol{j} \grave{o}=n \operatorname{di} \int \boldsymbol{u}=\mathbf{j o ̀}$
DEM.SG=olla $=$ DEM.SG 3.PST-romper
DEM.PL $=$ mujer=DEM.PL
'Esa olla la rompieron esas señoras.' [elcd.ord]

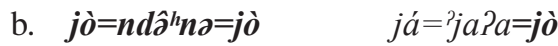
DEM.PL=flor=DEM.PL $\quad$ PTL=IMP.podrir=DEM.PL
'Ya se pudrieron estas flores.' [txt.diag]

Por último, jò da cuenta de una relación gramatical, objeto en (26a) y sujeto en (26b); esto ocurre si este demostrativo aparece como cierre oracional.
a. $n \grave{u}=6 \grave{z} z o ́$
yú-fát'ì $=\mathbf{j o ̀}$
ART.DEF.SG=hombre 3.PST-raspar=DEM.PL
'El hombre raspó esos.' [txt.nar] 
b. $\quad$ ú$^{\prime}-m b \check{P} ว=\mathbf{j o ̀}$

3.PST-vender=fruta.DEM.PL

'Se vendió esas (frutas).' [txt.nar]

En conversaciones, narraciones, descripciones y diálogos se usa el demostrativo plural $k^{\prime} \grave{o}$. Esta forma se enclitiza en el nominal, como en (27). Esta forma enclítica está asociada con la distancia de lejanía que presenta la entidad expresada dentro del discurso. En los ejemplos de (27) k'òfeyo 'esa manteca' y k'òth'úhitbe 'esos bancos de nosotros dos', los referentes de estas entidades no están presentes dentro del espectro del hablante, y ya han sido nombrados con anterioridad dentro del discurso.
a. $n \grave{u}=m \check{\partial} h \dot{m}$
ø-po?o
$\boldsymbol{k}^{\prime} \boldsymbol{o}=\int \boldsymbol{e y} \boldsymbol{\jmath}$
ART.DEF.SG=plato
3.PRS-tener
DEM.PL $=$ manteca
'El plato tiene esa manteca.' [elcd.lxc]
b. $\quad$ й $=h m \tilde{n} \tilde{o}$
yú-sà?a
$\boldsymbol{k}^{\prime} \grave{o}^{\prime}=\boldsymbol{t}^{h} \boldsymbol{u}^{h} \boldsymbol{h} \boldsymbol{i}=\mathbf{b e}$
ART.DEF.SG=coyote 3.PST-estar.mordiendo DEM.PL-1.POs.banco=DL.EXCL
'El coyote estuvo mordiendo estos bancos de nosotros dos.' [txt.el coyote]

De igual manera, el demostrativo plural k'ò aparece como cierre oracional expresando una relación gramatical de objeto directo, como en (28a); o se enclitiza en el nominal, si la FN en la que aparece está modificada por un numeral, como en (28b).
a. $j \grave{o}=n d i f u$

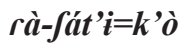
ART.DEF.PL $=$ mujer $\quad 3$. FUT-raspar $=$ DEM
'Las mujeres rasparán eso.' [txt.nar]

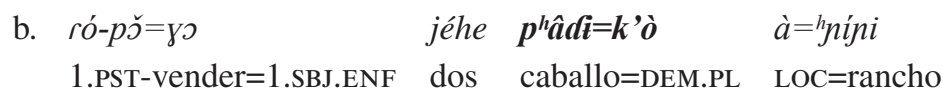
'Vendí estos dos caballos en el rancho.' [elcd.ord]

La diferencia entre artículos y demostrativos está determinada por la posición de estos dentro de la frase nominal y de la oración: el artículo aparece al inicio de la FN y como cierre de oración, pero no como cierre de FN. Por su parte, 
un demostrativo aparece al inicio de la FN, como cierre de FN y como cierre de oración. La forma reducida de los demostrativos que están en posición preverbal, se enclitiza en el verbo y en la frase nominal que se encuentre al final de la oración; un artículo no aparece en estos contextos.

En suma:

a) Los artículos aparecen al inicio de la FN y como cierre de la oración, pero nunca como cierre de la FN.

b) Los artículos son siempre proclíticos (a menos que funcionen como cierre oracional).

c) Los artículos no cambian nunca su tono (a menos que se trate de la tercera persona posesiva).

d) Los artículos coaparecen con cualquier marca de plural.

e) Los demostrativos pueden aparecer al inicio de la FN, como cierre de la FN y como cierre de la oración.

f) Los demostrativos como morfemas libres nunca cambian el tono.

g) Los demostrativos en sus formas reducidas, sin embargo, pueden o no cambiar el tono.

h) Los demostrativos no coaparecen con las marcas de plural.

\section{Numerales}

Los numerales náha 'uno', jéhe 'dos', hnîi 'tres', tshì?o 'cuatro' modifican a los nominales, como en (29). El artículo definido solo coaparece en la FN con el numeral náha 'uno', como en (29a); con otros numerales, como en (29b), no es posible que coocurran con artículos. Los nominales modificados por numerales se ubican en diferentes posiciones dentro de la oración, como en (29c) y (29d).
a. rí-fŏsì
náha nù=mífi
1.PRS-levantar uno ART.DEF.SG-gato
'Levanto un gato.' [elcd.lxc]
b. *nù=t'îi yú-pวัРna jéhe jo=ygùmi ART.DEF.SG=niño
3.PST-robar dos ART.DEF.PL $=$ casa
'El niño robó dos casas.' [elcd.lxc]



c. ไníi i-ni'je ró-mă?ma
tres 1.POS-dedo 1.PST-lastimar
'Me lastimé tres dedos.' [elcd.lxc]
d. zú-j'òrì tshì̀o péso
3.PST-pedir cuatro peso
'Pidió cuatro pesos.' [elcd.lxc]

El demostrativo plural $n \grave{u} h j o ̀$ aparece en FN modificadas con numerales, como en (30a); este se ubica antes o después del numeral, como en (30b); la forma reducida del demostrativo plural jò, como en (30b), aparece como cierre de la oración, pero no se puede proclitizar en el numeral, como en (30c).
a. è-hòsé yú-thitsi
nùhjò jéhe ygùmi=jò
NP-José 3.PST-limpiar
DEM.PL dos
casa=DEM.PL
'José limpió estas dos casas.' [txt.diag]
b. è-hòsé yú-thitsi jéhe nùhjò=ngùmi=jò
NP-José 3.PST-limpiar dos DEM.PL=casa=DEM.PL
'José limpió estas dos casas.' [txt.diag]
c. *è-hòsé yú-thitsi jò=jéhe ygùmi
NP-José 3.PST-limpiar ART.DEF.PL $=$ dos casa
'José limpió (las) dos casas.' [txt.diag]

\section{Cuantificadores}

Los cuantificadores téfe 'todo', háygəntsi 'algunos', nà púnk’ì 'mucho' y t'íke ‘poco’ están ejemplificados de (31) a (35). La FN en donde aparece el cuantificador téfe 'todo' coaparece con el artículo definido plural jò, como en (31). Si una FN con este cuantificador se antepone al verbo, aparece la marca de plural $h i$, enclitizada en el verbo. En los ejemplos de (31b) y (31c), el enclítico hi hace correferencia con la frase nominal que expresa al sujeto.
a. $\quad$ zú-p̌̌Pna
té
3.PST-robar todo ART.DEF.PL=blanco-pollo
'Se robaron todos los pollos blancos.' [txt.diag] 

b. téfe jòn=t'í?i
ø-ná $=\boldsymbol{h i}$
$\grave{e}-\int u ́ \beta a$
todo ART.DEF.PL=niño 3.PRS-hablar=PL NP-Juan
'Todos los niños hablan con Juan.' [txt.diag]

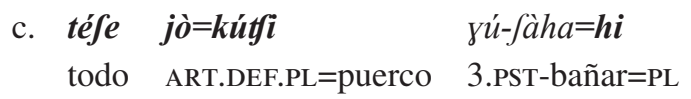

'Todos los puercos se bañaron.' [elcd.cuant]

Igualmente, una FN modificada por el cuantificador téfe 'todo' coaparece con el demostrativo plural $[j \grave{o}==j \grave{o}]$ y la forma reducida de este demostrativo jò, como en (32); este cuantificador se ubica en la parte más externa de la FN, como en (32a), o dentro del ámbito del demostrativo, como en (32b). En estos casos no aparece en el verbo la marca de plural, $h i$.
a. téfe jò=kútfi=jò
yú-fàha
todo DEM.PL=puerco=DEM.PL 3.PST-bañar
'Todos esos puercos se bañaron.' [elcd.cuant]

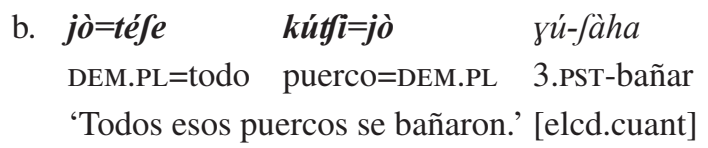

Háygontsi 'algunos', mostrado en (33), cuantifica los nominales nucleares; la FN con este tipo de cuantificador se puede mover dentro de la oración; este cuantificador no coaparece con ningún artículo o demostrativo, como tampoco ocurre la marca de plural, $h i$.
a. mí-nána
уú-Póti?i

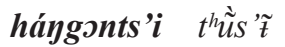
1.POS-mamá 3.PST-hacer algunos sombrero
'Mi mamá hizo algunos sombreros.' [elcd.ord]
b. rà- $\int \grave{o} s ’ \grave{t}$
háygonts'i fỉka thó? $\tilde{o}$
3.FUT-cargar algunos bulto maíz
'Cargará algunos bultos de maíz.' [elcd.cuant]
c. hángonts’i hmî́ñ è-fùßa rà-mb̀̀rì
algunos coyote NP-Juan 3.FUT-cuidar
'Juan cuidará algunos coyotes.' [elcd.cuant] 
El cuantificador nà púnk'í 'mucho', ilustrado en (34), igualmente aparece cuantificando la entidad que está expresada en una FN; no coaparece con ningún artículo o demostrativo. Las FN con este cuantificador se mueven dentro de la oración; la marca de plural hi no ocurre.
a. è-màría ø-hítì nà-púnk’i měrio
NP-María 3.PRS-tener PRED-mucho dinero
'María tiene mucho dinero.' [elcd.lxc]
b. è-fúßa yú-tǔhmi nà-púnki zá?a
NP-Juan 3.PST-sembrar PRED-mucho árbol
'Juan sembró muchos árboles.' [elcd.lxc]

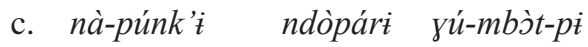
PRED-mucho zopilote 3.PST-matar-3.DAT
'Le mató muchos zopilotes.' [elcd.ord]

El cuantificador t'íke 'poco', mostrado en (35), presenta algunas restricciones semánticas. Este cuantificador no coocurre con entidades animadas, como en (35a) y (35b). Este cuantificador ocurre solo con núcleos nominales que denotan entidades inanimadas ejemplificadas de (35c) a (35e). Estas FN tienen la posibilidad de moverse al interior de la oración. Las FN con cuantificador ty'ike 'poco' no aparecen ni con artículos, ni con demostrativos; igualmente, la marca de plural hi no ocurre.
poco paloma 3.PST-comer maíz
'Pocas palomas se comieron el maíz' [elcd.cuant]
a. “ty'ike mbàro yú-sìî tốPõ

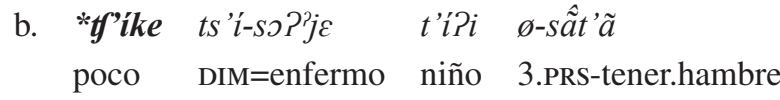
'Pocos niños enfermos tienen hambre' [elcd.cuant]
c. è-kárlo yú-kitsi tyike pèj’i
NP-Carlos 3.PST-cortar poco tejocote
'Carlos cortó poco tejocote.' [txt.diag]
d. ø-sißi ty'ike ndèmófi
3.PRS-sacar poco jitomate
'Saca un poco de jitomate.' [txt.diag] 


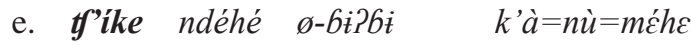

poco agua 3.PRS-haber LOC=ART.DEF.SG=pozo

'Hay poca agua en el pozo.' [txt.diag]

Las expresiones de cuantificación del tipo sàya jéhe 'los dos' o sàya ts hì?o 'los cuatro', como en (36) y (37), ocurren en FN en donde el nominal está modificado por el artículo definido plural jò. Las FN con este tipo de cuantificador se mueven dentro de la oración; la forma del plural hi ocurre en los contextos en donde la FN está en posición preverbal. La forma sàya ha sido glosada como delimitador, ya que solo coocurre con numerales y expresa un sentido de delimitación.
a. yú-t̀̀mi-hi
sàya j'éhe jò=phándehe
3.PST-comprar-PL DEL dos ART.DEF.PL=venado
'Compraron los dos venados.' [elcd.lxc]

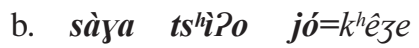 yú- ${ }^{h} w a ́ t i=h i$
DEL cuatro ART.DEF.PL $=$ falda 3.PST-romper $=$ PL
'Las cuatro faldas se rompieron.' [elcd.lxc]

Con estas formas de cuantificación, el numeral se reduce y se ubica entre el delimitador y la marca de plural, como en (37), sàya hnihi 'los tres' de (37a) y sàya j'éhi 'los dos' de (37b).
3.PRON.SG 3.PRS-jalar DEL tres=PL ART.DEF.PL=collar
'Ella jala los tres collares.' [elcd.lxc]
a. aygèze ø-hwintfi sàya hni=hi jò=thèße
b. sàya j’é=hi jò=mbókw ø- ${ }^{h}$ wátít
DEL dos=PL ART.DEF.PL=huarache 3.PRS-romper
'Los dos huaraches se rompen.' [elcd.lxc]

\section{Conceptos de propiedad}

En el sistema gramatical del mazahua los sustantivos se modifican por conceptos de propiedad que están formalizados en cláusulas relativas (una descripción 
amplia de los conceptos de propiedad se encuentra en Smith-Stark, 2004; Palancar, 2006; Villard, 2013). Los nominales están modificados por oraciones cuyos núcleos son verbos de estado y verbos incoativos. ${ }^{6}$ En los ejemplos de (38) a (41) los estados y los verbos incoativos son los núcleos verbales de las oraciones subordinadas. Los verbos de la cláusula relativa están flexionados en diferentes tiempos: presente, (38); pasado, (39); pasado inmediato, (40), y futuro, (41).
a. $n \grave{u}=6 \grave{c} z o$
$\varnothing \quad n \grave{a}-z \dot{\varepsilon} n j ’ i$

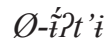
$n \grave{u}=r e ́ k^{w} a$
ART.DEF.SG=hombre
REL PRED.PRS-fuerte
3.PST-amarrar
ART.DEF.SG=burro
'El hombre (que es) fuerte amarró al burro.' [elcd.lxc]

${ }^{6}$ A continuación se presenta una lista de estados y verbos incoativos que modifican a los nominales:

Dimensión: hẽ̌Pẽ 'profundo', máha 'alto', ndáPa 'alto', tsíndù?ta 'chaparro', nòho 'grande', pidzi 'grueso', láyga '(muy) flaco', 'mas' $\dot{t}$ 'flaco', přpi 'gordo', mbìt 'ancho'.

RASGO FÍsICO: fini 'ruidoso', 'wïPì 'pesado', pêtfe 'pegajoso', péPe 'pegajoso', pófí 'sucio', $s$ 'jri 'sucio', j'à?a 'podrido', ph̀̀nt'a 'hinchado', $k^{h} \tilde{O}$ ? $\tilde{o}$ 'amargo', bèj'e 'mojado (por la lluvia)', ká?a 'mojado (por alguien)', rándzo 'nuevo', hว̌Pっ 'sa-

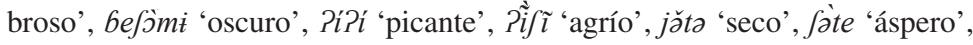

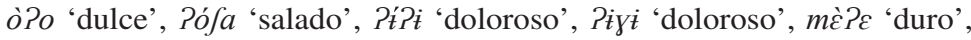
tònga 'débil', Púmbi 'rápido', tsákwana 'rápido', páPa 'caliente', hôti 'frío',

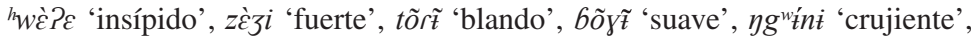

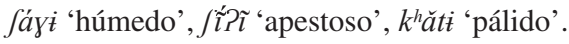

Color: $\quad$ hjásí 'claro' (la luz del día), jorì 'claro' (el agua), mbèze 'verde', $k$ 'àft’ 'amarillo', kã̀ygã 'morado', pòthi 'negro', mbàha 'rojo', t’’́fít 'blanco'.

Propensión

HUMANA: SóPo 'bonita, ser guapo', s'óPo 'malo', s'óPo 'grosero', 'já-sóPo 'feo', ndúntu 'tonto' (animal), fìngo 'tonto' (humano).

Evaluación: hóPo ‘bueno’, tsómbene ‘bueno’ (humano), fénse ‘bueno'(alimentos).

VALOR:

minfi 'caro'.

RASGO

PSICOLÓGICO: Gíbo 'inteligente', sélo 'celoso', Pòmbì 'tramposo', bètsa 'travieso', mbغ̀ $p^{h} i$ 'trabajador', tì̀rî 'borracho', ygòne 'mudo', ygòyo 'sordo', hjòja 'huérfano', mbذ̀yi 'tierno', màha 'contento'.

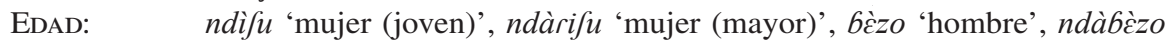
'hombre mayor', zìt'a 'viejo' (humano), ż̀ेe 'viejo'. 

b. $n \grave{u}=k^{h} \grave{i}^{h} n \dot{t}$
$\varnothing \quad$ nà-péPe

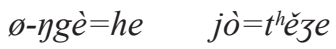
ART.DEF.SG $=$ masa REL PRED.PRS-pegajoso
3.PRS-Ser=PL ART.DEF.PL=tamal
'La masa (que es) pegajosa es para los tamales.' [elcd.lxc]

(39)

a. nù=fút $i \quad \varnothing \quad$ mà-sóro

ART.DEF.SG=niña REL PRED.PST-bonita 3.FUT-lavar ART.DEF.3.POS.PL=ropa

'La niña (que estaba) bonita lavará su ropa.' [elcd.lxc]
b. è-hòsé yú-tòmì nù=hnôna
NP-José 3.PST-comprar ART.DEF.SG-comida REL PRED.PST-salada
'José compró comida (que era) salada.' [elcd.lxc]

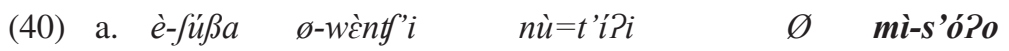

NP-Juan 3.PRS-regañar ART.DEF.SG=niño REL PRED.PST-malo

'Juan regaña al niño (que estaba) grosero.' [elcd.lxc]

b. rá-dà nù=hnôna

1.FUT-dar ART.DEF.SG=comida REL PRED.PST-caliente

'Le daré la comida (que estaba) caliente.' [txt.diag]

(41)
a. è-màría rà-pàjât i-ndzùmí $\varnothing \quad$ rà-nóho
NP-María 3.FUT-barrer 1.POS-casa REL PRED.FUT-grande
'María barrerá nuestra casa (que será) grande.' [txt.diag]
b. $n \grave{u}=t$ ' $\imath ? i$
$\varnothing \quad g \grave{a}-n d \check{a} P a$
ø-クgèhi
t'i?i
ART.DEF.SG-niño REL PRED.FUT-alto 3.PRS-ser.1.POS hijo
'El niño (que será) alto es mi hijo.' [elcd.lxc]

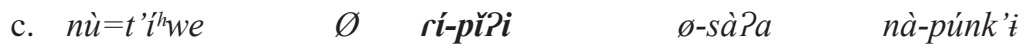
ART.DEF.SG=niña REL PRED.FUT-gordo 3.PRS-comer PRED-mucho
'La niña (que estará) gorda come mucho.' [elcd.lxc]

Las construcciones que expresan conceptos de propiedad pueden o no presentar un relativizador o pronombre relativo. Antes de continuar es necesario mencionar que la lengua no dispone de un paradigma de relativizador o pronombre relativo; en su lugar, se utiliza la estrategia de los artículos o demostrativos; en el caso de los ejemplos anteriores no aparece ningún artículo o demostrativo en función de relativizante $(\varnothing)$. 
En (42) el artículo nù aparece en función de relativizante; por razones prácticas, a este tipo de artículo o demostrativo se lo ha glosado como 'relativizador'. Generalmente, el 'relativizador' es la misma forma del artículo que está modificando al sustantivo nuclear: nù y jò.
a. $n \grave{u}=t^{\prime} \grave{\imath} i$
nù
mà-tfáso?

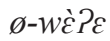
k'ó=nú-nána
ART.DEF.SG-niño REL.SG PRED.PST=feo 3.PRS-llorar COM-ART.DEF.3.POS.SG-mamá 'El niño feo llora con su mamá.' [elcd.lxc]
b. $j \grave{o}=n d \hat{\partial}^{h} n \partial$
jò
nà-sò?o : yú-j’òti
ART.DEF.PL=flor REL.PL PRED.PRS=bonita 3.PST-secar
'Las flores bonitas se secaron.' [txt.diag]

Otro recurso que presenta la lengua para modificar a los nominales es el de la nominalización (Givón, 2001), como en (43). Las frases modificadoras están formadas por un núcleo que expresa un estado y este está modificado por un artículo; en (43), las dos FN están yuxtapuestas.
a. nùtskh ró-ṫ̀mì
nù=ránḑo
nù= $=$ gùmi
1.PRON 1.PST-comprar ART.DEF.SG=nuevo
ART.DEF.SG=casa
'Yo compré la casa nueva.' [elcd.ord]

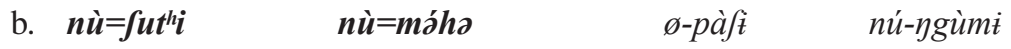
ART.DEF.SG=niña ART.DEF.SG=contenta 3.PRS-barrer ART.DEF.3.POS.SG-casa
'La niña contenta barre su casa.' [elcd.lxc]

Las modificaciones nominales de (44) están formadas por un nominal que denota un estado y un solo artículo que modifica a toda la FN; en este tipo de FN la unidad léxica que modifica al núcleo nominal se ubica antes o después del nominal.
a. nù=ndój'e
'jó?o ø-sât' $\tilde{a}$
ART.DEF.SG-flaco perro 3.PRS-tener.hambre
'El perro flaco tiene hambre.' [txt.diag] 
b. jò=té?e ts'ómbéne ø-zéng ${ }^{w} a \quad j o ̀=t ' i ́ P i$

ART.DEF.PL=gente amable 3.PRS-saludar ART.DEF.PL-niño

'La gente amable saluda a los niños.' [elcd.lxc]

\section{Compuestos}

Los compuestos están formados por una unidad léxica que denota un estado y un sustantivo, ${ }^{7}$ como en (45). Pueden o no estar modificados por un artículo y se pueden mover dentro de la oración.
a. $n \grave{u}=n d \grave{\jmath} f u$
rà-Pókì
$n \grave{u}=z \grave{\varepsilon}-p^{h \grave{\imath} n k^{w} a}$
ART.DEF.SG=mujer 3.FUT-cortar ART.DEF.SG=viejo-petate
'La mujer va a cortar el petate viejo.' [elcd.lxc]

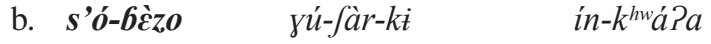
malo-hombre 3.PST-bañar-1.DAT 1.POS-conejo
'El hombre malo bañó a mi conejo.' [elcd.lxc]

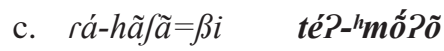
1.FUT-asar=DL crudo-pescado
'Nosotros dos asaremos el pescado crudo.' [elcd.lxc]

La FN que expresa al compuesto ocurre con un cuantificador, un numeral o un prefijo de dimensión, como en (46).

7 Estados que aparecen en unidades léxicas compuestas:

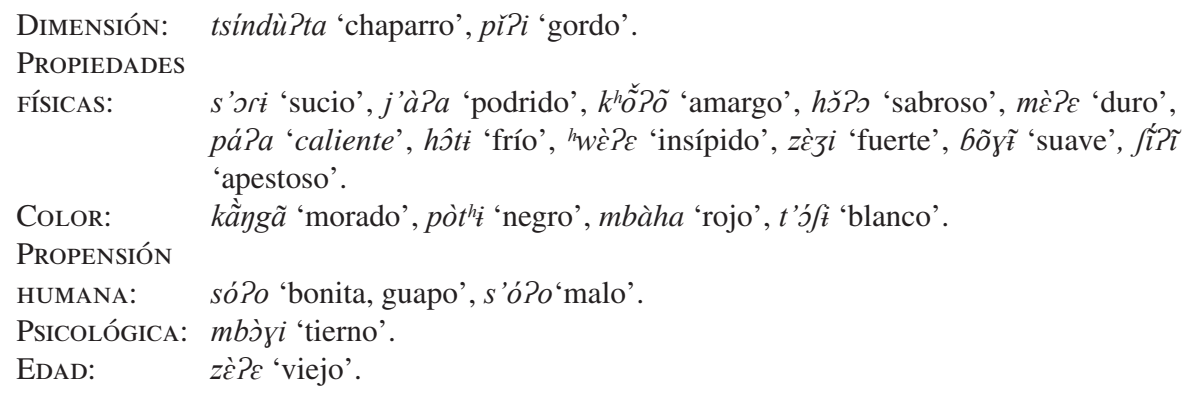


(46)

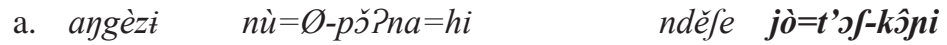
3.PRON.PL ART.DEF.SG=3.PST-robar=PL todos ART.DEF.PL=blanco-pollo
'Ellos robaron todos los pollos blancos' [elcd.cuant]
b. nùtsk ${ }^{h}$ ró-néPe jéhe póth $\boldsymbol{k}^{\boldsymbol{h}} \boldsymbol{h}$
1.PRON.SG 1.PST-morder dos negro-hongo
'Yo mordí dos hongos negros' [elcd.lxc]

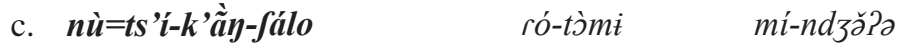
ART.DEF.SG=DIM-morado-jarro 1.PST-comprar TADV-ayer
'El jarrito morado lo compré ayer' [elcd.lxc]

\section{Cláusulas relativas}

Las oraciones relativas que modifican a los nominales son de dos tipos: núcleo externo y núcleo interno (Keenan, 1985; Andrews, 2007). Las del primer grupo están ilustradas en (47) y (48) y las del segundo en (49) y (50). Contrario a las relativas que expresan conceptos de propiedad, ejemplificadas de (38) a (42), los sustantivos están modificados por oraciones relativas que están introducidas, por una parte, por los artículos singulares k'á y nù $\mathrm{y}$, por otra, por el demostrativo plural k’ó y el artículo plural jò.
$\varnothing-p \check{P}$
$n \grave{a}=n d \hat{c} n t^{h} \grave{i}$
k'á já=mì-néPe
1.PRON.SG 1.PST-vender ART.INDF=borrego REL.SG NEG=PSTR-comer nà-h̆ĥna
a. nùtsk's
ART.INDF-comida
'Vendí un borrego que no quería comer.' [txt.diag]

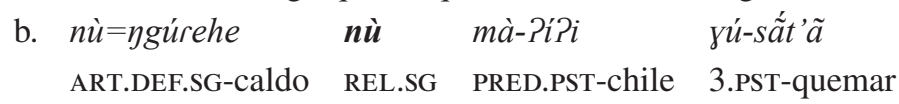
'El caldo que estaba picoso se quemó.' [elcd.ord]
a. $\quad \grave{e}-\int u ́ \beta a \quad ø-s i ̀ i$
$j \grave{o}=n d \grave{f} / i$

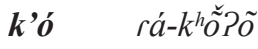
NP-Juan 3.PRS-comer ART.DEF.PL=naranja REL.PL PRED.FUT-amargo
'Juan se come las naranjas que estarán amargas.' [elcd.lxc] 

b. $n \grave{u}=6 \grave{\varepsilon} z o$

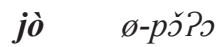
$6 a ? a$
$\varnothing-h \hat{t} P t \tilde{t}=h i \quad n \grave{u}=6 a ́ y a$

ART.DEF.SG=hombre REL.PL 3.PRS-venderleche 3.PST-amarrar=PL ART.DEF.SG=vaca 'Los hombres que venden leche amarraron a la vaca.' [elcd.ord]

En (49a), el artículo singular k'á es el núcleo de la oración relativa, mientras que en (49b) esta función la realiza el demostrativo plural k'ó. En las construcciones relativas de núcleo interno no son posibles los artículos nù y jò, de las relativas de núcleo externo, como en (49c).
a. $\quad \boldsymbol{k}^{\prime} \boldsymbol{a} \quad n \grave{u}=\eta g u ̀ m m i$
Ø-hàtว
ø-tîfi
nà-púnk'ì
REL.SG ART.DEF.SG=casa
3.PST-construir 3.PRS-gotear
PRED-mucho
'La casa que construyeron gotea mucho.' [txt.diag]
b. k'ó $n \grave{u}=s^{\prime} \partial 6 \partial$ ró-wánt't
$\varnothing$-jìri
mì-nỏ̧á?a
REL.SG ART.DEF.SG=olla 1.PST-enjuagar 3.PST-romperse TADV-ayer
'La olla que enjuagué se rompió ayer.' [elcd.lxc]

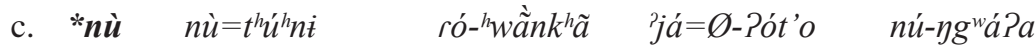
REL.SG ART.DEF.SG=silla 1.PST-sacudir NEG-3.PRS-tener ART.DEF.3.POS.SG-pie 'La silla que sacudí no tiene patas.' [elcd.lxc]

\section{Posesión nominal}

La posesión nominal se compone por un núcleo nominal y un prefijo; la posesión se codifica de manera simultánea en el prefijo con tono. Los prefijos se muestran en el Cuadro 5. La entidad poseedora se exhibe en los prefijos con tono y la entidad poseída se expresa en el núcleo nominal (Mora-Bustos \& Mora Muñoz, 2016). ${ }^{8}$

En (50), tanto el prefijo de la primera persona singular ín, de (50a), como el prefijo de la segunda persona singular ìn, de (50b), son formas segmentales idénticas; se distinguen únicamente por sus rasgos tonales.

8 El concepto de posesión ha sido tratado por diferentes autores, entre ellos se han retomado a Seiler (1983) y Aikhenvald y Dixon (2013). 


\begin{tabular}{|c|c|c|}
\hline PeRsona & SINGULAR & PLURAL \\
\hline 1 & ín- & $\begin{array}{l}\text { jò=ín- } \\
\text { nùnjó́=ín- }\end{array}$ \\
\hline 2 & ìn- & $\begin{array}{l}\text { jò=ìn- } \\
\text { nùnjó=in- }\end{array}$ \\
\hline 3 & $\begin{array}{l}\text { nú- } \\
\text { nùnú- }\end{array}$ & $\begin{array}{l}\text { jó- } \\
\text { nù̀jó- } \\
\text { k'ó- } \\
\text { nùnjó=jó- }\end{array}$ \\
\hline
\end{tabular}

(50) a. è-pèyro yú-sì̀i ín-thómety'i

NP-Pedro 3.PST-comer 1.POS-carne

'Pedro se comió mi carne.' [elcd.lxc]

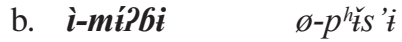

2.POS-corazón 3.PRS-palpitar

‘Tu corazón palpita.' [elcd.lxc]

El recurso lingüístico que dispone la lengua para expresar la pluralidad de los poseedores consiste en la coaparición de un artículo definido plural, como en (51), o de un demostrativo plural, como en (52). Los prefijos y los tonos que exhiben al poseedor no presentan ningún tipo de variación con respecto a los codificados en la frase posesiva singular.

a. nú-’’jópo

$\varnothing-p h \dot{\partial r i}$

jò=in $\mathbf{t} \boldsymbol{t}^{h} \grave{\partial} h \boldsymbol{\partial}$

ART.DEF.3.POS.SG-perro 3.PRS-cuidar

ART.DEF.PL $=1$. POS-surco

'Su perro cuida mis surcos.' [elcd.lxc]

b. Jú-tsh̆̌nts'i nù=ndàrehe jò=ì-ndzôni

3.PST-ahogar ART.DEF.SG=río ART.DEF.PL=2.POS-pollo

'El río ahogó tus pollos.' [txt.diag]

9 La versión sobre la posesión que se presenta en este trabajo es mucho más sintética que la desarrollada en Mora-Bustos y Mora Muñoz (2016). 


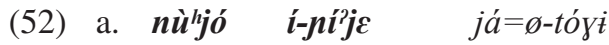

DEM.PL 1.POS-dedo PTL=3.PRS-cortar

'Mis dedos se cortaron.' [elcd.lxc]

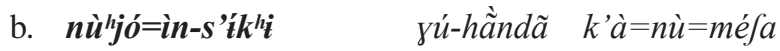

DEM.PL=2.POS.SG-arete 3.PST-ver LOC=ART.DEF.SG=mesa

'Vi tus aretes en la mesa.' [txt.diag]

La tercera persona, singular y plural, presenta un mayor número de formas para codificar la entidad poseída, esto es, el artículo definido singular o plural, como en (53), y el demostrativo singular y plural, como en (54). En los artículos y demostrativos se configura un tono alto para dar cuenta de la posesión; estos proclíticos presentan un tono bajo en los contextos en donde no se codifica la posesión.

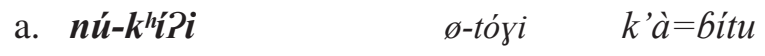

ART.DEF.3.POS.SG-Sangre 3.PST-caer LOC=ropa

'Su sangre cayó en la ropa.' [elcd.lxc]

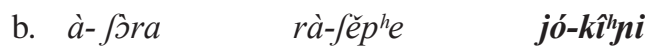

TADV-mañana 3.FUT-cosechar ART.DEF.3.POS.PL-nopal

'Mañana cosechará sus nopales.' [elcd.ord]
a. aygèze yú-thitsi nùnú-jók'i
3.PRON.SG 3.PST-limpiar DEM.3.POS.SG-vómito
'Él limpió su vómito.' [txt.diag]
b. $j a ́=\varnothing-j j a ? a \quad \boldsymbol{n u ̀ ̆ h o ́ ~}$
PTL=3.PST-podrirse DEM.3.POS.PL flor
'Ya se pudrieron sus flores.' [elcd.ord]

Las relaciones de parentesco se caracterizan por contar con un sistema de prefijos de posesión propio, como se muestra en el Cuadro 6.

En (55) se ejemplifican los prefijos de posesión de parentesco (Mora-Bustos \& Mora Muñoz, 2016). 
Cuadro 6. Prefijos de posesión en relaciones de parentesco

\begin{tabular}{clll}
\hline PERSONA & \multicolumn{2}{c}{ SINGULAR } & PLURAL \\
\hline 1 & mí- & ín- & jò=ín- \\
2 & mì- / ní- & ìn- & jò=ìn- \\
3 & nú- & nú- & jó- \\
\hline
\end{tabular}

(55)

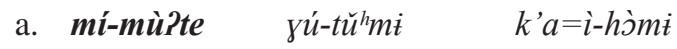

1.POS-cuñada 3.PST-sembrar LOC=2.POS-tierra

'Mi cuñada sembró en tu tierra.' [elcd.lxc]

b. mì-kwărma

2.POS-hermano 3.PRS-raspar-3.DAT LOC=maguey

'Tu hermano te raspa por el maguey.' Lit.

'Tu hermano te raspa por el sendero en donde se encuentra el maguey.' [elcd.lxc]

c. rá-nème kò nú-běj’i

1.FUT-bailar COM ART.DEF.3.POS.SG-pariente

'Yo bailaré con su pariente.' [txt.diag]

Las entidades de parentesco no tienen formas propias para codificar el singular y el plural de tercera persona; de la misma manera, las formas posesivas de la primera y segunda persona retoman el sistema de posesión general, como en (56).
a. nú-t'í?i
yú-pà?a $\quad k^{\prime} \grave{a}=n i t$ himi $^{\prime}$
ART.DEF.3.POS.SG-hijo 3.PST-ir LOC=iglesia
'Su hijo fue a la iglesia.' [txt.diag]
b. jòn=in-ndzâ?a
ø-pว̌ว

$$
\text { jò=t'ěne }
$$
ART.DEF.PL=1.POS-suegro 3.PRS-vender ART.DEF.PL=juguete
'Mis suegros venden juguetes.' [elcd.tam]
c. $j \grave{j}=\grave{i n}-\boldsymbol{k}^{w}$ ărma

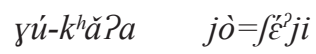
ART.DEF.PL=2.POS-hermano 3.PST-hacer ART.DEF.PL=tortilla
'Tus hermanos hacen tortillas.' [txt.las tortillas]
d. jó-ménche
уù-?ё̌he
ART.DEF.3.POS.PL-yerno 3.PST-venir
'Sus yernos vinieron.' [txt.diag] 


\section{Frase posesiva compleja: relaciones no eventivas}

Las relaciones semánticas se dan entre dos entidades, cada una de las cuales puede ser animada, inanimada, una locación, o bien, una relación social (Ortiz, García, Mora-Bustos \& Arellanes, 2014). Los dominios considerados en la propuesta de estos autores son cuatro: a) Dominio material 1: parte-todo (partes del cuerpo), secreción/excreción del cuerpo, posesión inalienable, posesión alienable; b) Dominio material 2: parte-todo (partes de las entidades inanimadas), contenedor-contenido actual, porción-todo, contenedor-contenido potencial; c) Dominio locativo: tema-locación fija, tema-locación temporal, tema-origen; d) Dominio social: parentesco intergeneracional (incluido el matrimonio), parentesco transgeneracional, otras relaciones sociales. En mazahua el recurso gramatical para codificar estas relaciones semánticas son la prefijación con tono y la yuxtaposición (Mora-Bustos \& Mora Muñoz, 2016).

Las entidades que son parte del dominio material 1 utilizan la prefijación con tono para codificar la posesión, como se observa en los ejemplos de (57) a (59). Las relaciones de posesión inalienable en (60) están yuxtapuestas.

(57) Parte-todo (partes del cuerpo)
a. $\quad$ zú-h̀̀-pí
$n u ́-j$ 'ìzi
$n \grave{u}=6 \grave{\varepsilon} z o ́$
3.PST-curar-3.DAT
ART.DEF.3.POS.SG-cuello
ART.DEF.SG=hombre
'Le curó el cuello al señor.' [elcd.lxc]

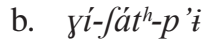
jó- thàngi
2.PST-raspar-3.DAT ART.DEF.3.POS.PL-pierna
$n \grave{u}=t^{\prime} i \hat{\imath} i$
ART.DEF.SG=niño

'Tú le raspaste las piernas al niño.' [elcd.ord]

(58) Secreción/excreción del cuerpo
a. aygéze jú-thitsi
$n u^{\prime}-k^{h} i \imath i$

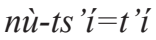
3.PRON 3.PST-limpiar ART.DEF.3.POS.SG-sangre
ART.DEF.SG-DIM=niño
'Él limpió la sangre del niñito.' (elcd.dime)
b. nù=mbáne
ø-s'ว́?
nú-titifi 'El bebé bebe la leche de la mujer.' [elcd.ord]
$n \grave{u}=n d \grave{l} f u$
ART.DEF.SG=bebé 3.PRS-beber ART.DEF.3.POS.SG-leche.materna ART.DEF.SG-mujer 
(59) Posesión inalienable
a. nùtsk'e ø-pant-mba
$n \dot{u}-t y \imath^{h} m \tilde{\imath}$
$n \grave{u}=n d \xi \hat{\imath} n \dot{n}$
2.PRON.SG 3.PRS-golpear-3.DAT ART.DEF.3.POS.SG-pecho ART.DEF.SG=buey
'Tú le golpeas el pecho al buey.' [elcd.ord]

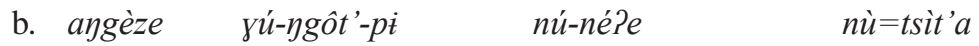
3.PRON.SG 3.PST-cerrar-3.DAT ART.DEF.3.POS-boca ART.DEF.SG=anciano 'Él cerró la boca del anciano.' [elcd.ord]

(60) Posesión alienable
a. yí-ngíi $i \quad n \grave{u}=6 o n e$
$n \grave{u}=n d \grave{l} f u$
2.PRS-tener ART.DEF.SG=machete ART.DEF.SG=mujer
'Tú tienes el machete de la señora.' [elcd.lxc]
b. rá-sất' $\tilde{a}=\beta i$
jò=ndzá?a
$n \grave{u}=6 \grave{\varepsilon} z o$
1.FUT-quemar=DL ART.DEF.PL=leña ART.DEF.SG=hombre
'Vamos a quemar las leñas del señor.' [elcd.ord]

La prefijación con tono es el recurso morfosintáctico que da cuenta de las relaciones del dominio social, ilustrado de (61) a (63). El grado de proximidad o cercanía que se establece en las entidades pertenecientes a este dominio es muy alto, ya que las relaciones de parentesco implican que los dos participantes involucrados se definan uno con respecto al otro ( $c f$. Ortiz et al., 2014).

(61) Parentesco intergeneracional
a. nú-bèt $t^{h} e ́$
nù=mámáyande
$\varnothing-$ - $\partial ̀ r$
$n \grave{u}=t^{\prime} \grave{\imath} \hat{\imath} i$
ART.DEF.3.POS.SG-nieto
ART.DEF.SG=abuela
3.PRS-oír
ART.DEF.SG=niño
'El nieto de la abuela escucha al niño.' [elcd.lxc]
b. nú-pápáyande

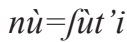 rà-?’̀rì
$\left.t^{h} \tilde{O}\right)^{\prime} \tilde{O}$
ART.DEF.3.POS.SG-abuelo ART.DEF.SG=niña 3.FUT-Pedir maíz
'El abuelo de la niña pedirá maíz.' [elcd.lxc]

(62) Parentesco transgeneracional
a. nú-nána
$n \grave{u}=n d \grave{\jmath} u$
ชú-wèे?
ART.DEF.3.POS.SG-mamá
ART.DEF.SG $=$ mujer
3.PST-1lorar
'La mamá de la mujer lloró.' [elcd.lxc] 

b. aygèze
$\varnothing-\int a ̀ t i$
nú-tá?a
$n \grave{u}=s \dot{j}^{P} j \varepsilon$
3.PRON.SG
3.PRS-bañar
ART.DEF.3.POS.SG-papá
ART.DEF.SG=enfermo
'Ella baña al papá del enfermo.' [elcd.ord]

(63) Otras relaciones de parentesco
a. nù-ygúne
$n \grave{u}=n d \grave{\imath} f u$
yú-ggitsi nù=lúlu
ART.DEF.3.POS.SG-comadre ART.DEF.SG=mujer 3.PST-cortar ART.DEF.SG=fruta
'La comadre de la mujer cortó la fruta.' [elcd.ord]

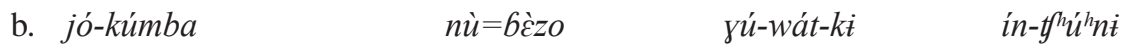
ART.DEF.3.POS.PL-compadre ART.DEF.SG=hombre 3.PST-romper-1.DAT 1.POS-banco
'Los compadres del hombre me rompieron mi banco.' [elcd.lxc]

Las entidades que forman parte del dominio material 2, como se muestra de (64) a (66), codifican la relación de posesión a través de la yuxtaposición. Solo en la relación parte-todo (entidades inanimadas) de (64) ocurre, además de la yuxtaposición, como en (64a), la prefijación con tono, como en (64b) y (64c).

(64) Parte-todo (entidades inanimadas)

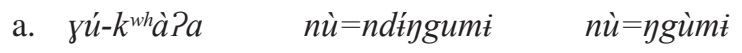

3.PST-terminar ART.DEF.SG=techo ART.DEF.SG=casa

'Terminaron el techo de la casa.' [txt.diag]

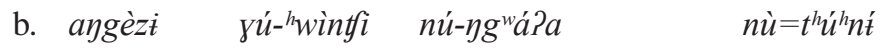

3.PRON.PL 3.PST-jalar ART.DEF.3.POS.SG-pie ART.DEF.SG=silla

'Ellos jalaron la pata de la silla.' [elcd.ord]

c. rì-hã̀ndã nú-níii nù=t'éhe

2.FUT-ver ART.DEF.3.POS.SG-cabeza ART.DEF.SG=cerro

'Tú verás la punta del cerro.' [elcd.lxc]

(65) Porción-todo
a. $\varnothing-j a-k{ }^{\prime} i$
nà=púno
kí?i
3.PRS-dar-1.DAT ART.INDF.SG=puñado frijol
'Me da un puñado de frijol.' [elcd.ord] 

b. è-ре́уго уú-sì?i
nú-mbárende
$n \grave{u}=t^{h} \dot{s}^{\prime} m e t^{\prime} i$
NP-Pedro 3.PST-comer ART.DEF.3.POS.SG-rebanada
ART.DEF.SG $=$ pan
'Pedro se comió la rebanada de pan.' [elcd.ord]

(66) Contenedor-contenido potencial
a. rá-fós't
$n \grave{a}=6 a r \grave{i}$
$t^{\prime} a p^{h} \dot{t}$
1.FUT-destapar ART.INDF=botella alcohol
'Destaparé una botella de alcohol.' [txt.diag]
b. zú-Pذ̀ri nù=tsí-fálo t'èmme
3.PST-pedir ART.DEF.SG=DIM-jarro atole
'Pidió un jarrito de atole.' [elcd.tam]

Finalmente, en el dominio locativo, de (67) a (69), la relación entre dos entidades que están conectadas por el vínculo de inclusión que se establece entre una locación y una entidad asociada se codifica a través de la yuxtaposición. El nominal se expresa con su respectivo determinante y la locación por el clítico k’à o su alomorfo $\grave{a}$.

(67) Tema-locación fija
a. ró-pá?a nù=nitshimi à=ßóndo
1.PST-ir ART.DEF.SG=iglesia LOC=México
'Yo fui a la iglesia de México.' [txt.diag]

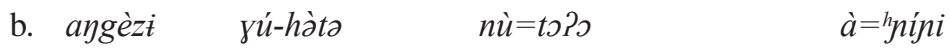
3.PRON.PL 3.PST-construir ART.DEF.SG=cementerio LOC=pueblo
'Ellos construyeron el cementerio del pueblo.' [txt.diag]

(68) Tema-locación temporal
a. zí-Pě้hẽ
$n \grave{u}=y \hat{\jmath}^{h} m a$
$k^{\prime} \grave{a}=n \grave{u}=e s k i n a$
2.PRS-venir ART.DEF.SG=mercado LOC=ART.DEF.SG=esquina
'Vienes del mercado de la esquina.' [elcd.lxc]
NP-María 3.PRS-trabajar ART.DEF.SG=tortillería enfrente
'María trabaja en la tortillería de enfrente.' [elcd.ord]

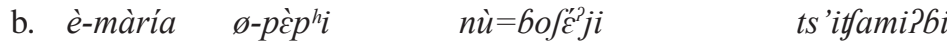


(69) Tema-origen
a. è-hosé rà-tĥt't
jò=mùru
$\grave{a}=$ san mateo
NP-José
3.FUT-encontrar
ART.DEF.PL=huevo
LOC=San.Mateo
'José encontrará huevos en San Mateo.' [txt.diag]
b. rá-hèj’i nù=mbèng ${ }^{w} a m a \quad \grave{a}=\int o^{h} w a$
1.FUT-rebanar ART.DEF.SG=camote LOC=Zitácuaro
'Voy a rebanar el camote de Zitácuaro.' [elcd.diag]

La prefijación con tono codifica relaciones de cercanía entre los poseedores y poseídos; igualmente, esta forma de codificación es obligatoria entre las entidades de las relaciones semánticas que no puedan ser concebidas sin el todo. La yuxtaposición codifica relaciones semánticas que expresen lejanía entre las entidades que constituyan parte de la relación. En las relaciones de cercanía solo entran los campos semánticos de partes del cuerpo de humanos, animales y algunos objetos, secreciones/excreciones de animales y personas, relaciones de parentesco, relaciones inalienables y algunos objetos alienables que varían de acuerdo con la concepción de dependencia de lo poseído con el poseedor. Las relaciones que no son intrínsecas, íntimas, inherentes u obligatorias se encuentran dentro del dominio material 2, esto es: materiales, líquidos, instrumentos de trabajo, partes de la casa, contenedores, alimentos, objetos dentro de la casa, instrumentos de cocina, ropa, accesorios, animales, verduras, frutas, plantas, entre otros. En el dominio de locación, al no establecerse una relación de posesión, en comparación con las entidades relacionadas en los otros dominios, se utiliza para su codificación una marca de locativo. Finalmente, en (70) se presenta el templete de la FN y en (71) se ilustra con algunos ejemplos.

(70) $($ CUANT-NUM $)=$ DEM/ART $=($ CUANTI/NUM $)=$ POS $<$ TON $>$-DIM-EST.SUST-SUST $=$ PL $=($ DEM $/$ ART) REL
a. sàya h̆hi=hi jò=ndênthì
nà-nòho
$k^{\prime} o ́$
$\varnothing-m b \check{2}$
DEL tres=PL ART.DEF.PL=borrego PRED.PRS-grande REL.PL 1.PST-vender
ø-sà?a s'aphari
3.PRS-comer zacate
'Los tres borregos grandes que vendí comen zacate' [elcd.ord] 


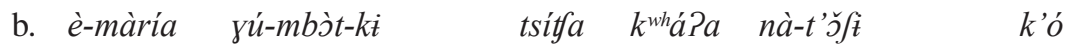 NP-María 3.PST-matar-1.DAT cinco conejo PRED.PRS-blanco REL.PL Ø-tómi
1.PST-comprar
'María me mató a cinco conejos blancos que compré '[elcd.lxc]
c. mí-nána rà-dà?a hníî mbàjo nà-máha k'ó Ø-hě̃ty'e 1.POS-mamá 3.FUT-dar tres rebozo PRED.PRS-largo REL.PL 3.PST-hilar 'Mi mamá dará tres rebozos largos que hiló' [elcd.lxc]

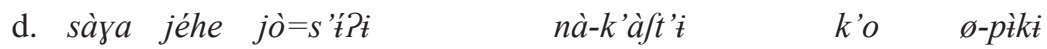
DEL dos ART.DEF.PL=pájaro PRED.PRS-amarillo REL.PL 3.PRS-picotear

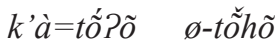
LOC=maíz 3.PRS-cantar

‘Los dos pájaros amarillos que picotean la milpa cantan’ [elcd.lxc]

\section{Conclusiones}

Sin haber mostrado un análisis minucioso y detallado, a lo largo de este trabajo se ha presentado una descripción de la estructura de la FN en mazahua. La FN es una unidad gramatical muy compleja en mazahua; está integrada por nominales sin ningún tipo de modificadores o por nominales con múltiples modificadores. Los nominales sin modificadores no aparecen en posición preverbal, para que esto ocurra deben estar modificados con los prefijos posesivos, un artículo, un determinante o coaparecer con un cuantificador. El sentido de dimensión del nominal está codificado en una serie de prefijos. La lengua presenta un sistema muy amplio para codificar el número: marcas de plural, artículos y demostrativos. El sistema de artículos y demostrativos es complejo, ya que está integrado por formas léxicas plenas y formas reducidas, las cuales se distribuyen en la FN o a lo largo de la oración. Algunos cuantificadores coaparecen en la FN junto a demostrativos, artículos y morfemas que codifican dimensión y posesión.

Los sustantivos son modificados por conceptos de propiedad que están formalizados en cláusulas relativas, esto es, los núcleos verbales de estas relativas son verbos de estado y verbos incoativos, los cuales se flexionan en diferentes tiempos; este tipo de modificación no presenta estrictamente un relativizador, la lengua dispone de la estrategia de pronombres y demostrativos. Las construcciones rela- 
tivas que modifican al nominal son de dos tipos: núcleo interno y núcleo externo; por lo general están introducidas por un gran repertorio de artículos y demostrativos. El sistema de posesión es muy amplio; la relación que establece la entidad poseedora con respecto a la entidad poseída se codifica en prefijos con tono; las relaciones semánticas no eventivas que se generan en los diferentes dominios semánticos se exhiben con prefijación con tono o a través de la yuxtaposición.

Finalmente, dado que se ha presentado una descripción panorámica de la estructura de la frase nominal, en estudios futuros sería conveniente elaborar descripciones monográficas de cada uno de los fenómenos gramaticales que hacen parte de una FN.

\section{Referencias}

Aikhenvald, Alexandra, \& Dixon, Robert M. W. (Eds.) (2013). Possession and ownership: A cross-linguistic typology. Oxford: Oxford University Press.

Amador Hernández, Mariscela (1976). Gramática del mazahua de San Antonio Pueblo Nuevo (Tesis de licenciatura, inédita). Escuela Nacional de Antropología e Historia, México.

Andrews, Avery D. (2007). Relative clauses. En Timothy Shopen (Ed.), Language typology and syntactic description: Complex constructions (2a. ed., Vol. 2, pp. 206-236). Cambridge: Cambridge University Press.

Dryer, MatThew S. (2007). Noun phrase structure. En Timothy Shopen (Ed.), Language typology and syntactic description: Complex constructions (2a. ed., Vol. 2, pp. 151-205). Cambridge: Cambridge University Press.

Dryer, Matthew S. (2013). Position of pronominal possessive affixes. En Martin Haspelmath, Matthew S. Dryer, David Gil \& Bernard Comrie (Eds.), The world atlas of language structures online. Leipzig: Max Planck Institute for Evolutionary Anthropology. Recuperado de http://wals.info/chapter/57

Dryer, Matthew S., \& Haspelmath, Martin (Eds.) (2013). The world atlas of language structures online. Leipzig: Max Planck Institute for Evolutionary Anthropology. Recuperado de http://wals.info.

Gibson, Lorna, \& Bartholomew, Doris (1979). Pame noun inflection. International Journal of American Linguistics, 45(4), 309-322.

Givón, Talmy (2001). Syntax: An introduction (Vols. 1 y 2). Ámsterdam: John Benjamins. 
Keenan, Edward L. (1985). Relative clauses. En Timothy Shopen (Ed.), Language typology and syntactic description: Complex constructions (Vol. 2, pp. 141-170). Cambridge: Cambridge University Press.

KnAPP Ring, Michael Herbert (2008). Fonología segmental y léxica del mazahua. México: Instituto Nacional de Antropología e Historia.

Knapp Ring, Michael Herbert (2013). Doctrina y enseñança en la lengua maçahua: estudio filológico y edición interlineal. Seguidos de un esbozo gramatical. México: Instituto Nacional de Lenguas Indígenas.

Kroeger, Paul R. (2005). Analyzing Grammar. An introduction. Cambridge: Cambridge University Press.

López Marín, ANTONio (2002). Estructura de la frase nominal en el jnatjo (mazahua) de la zona norte (Tesis de maestría). Centro de Investigaciones y Estudios Superiores en Antropología Social, México.

Mora-Bustos, Armando, \& Mora Muñoz, Gabriela (2016). El sistema de posesión en mazahua de Michoacán. Anales de Antropología, 50(2), 303-326.

Ortiz Villegas, Alejandra Itzel; García Zúñiga, Antonio; Mora-Bustos, Armando, \& Arellanes Arellanes, Francisco (2014). Syntax of the non-eventive semantic relations in four Otomanguean languages: Amuzgo, Mazahua, Mazatec and Zapotec. En 6th International Conference Syntax of the World's Languages. Pavía: Italia.

PAlAncAR, EnRIQUe L. (2006). Property concepts in Otomi: A language with no adjectives. International Journal of American Linguistics, 72(3), 325-366.

Palancar, EnRique L. (2009). Gramática y textos del hñoñho otomí de San Ildefonso Tultepec, Querétaro. México: Plaza y Valdés.

Plank, Frans (Ed.) (2003). Noun Phrase structure in the languages of Europe. Berlín: Walter de Gruyter.

Plank, Frans; Bossong, George, \& Comrie, Bernard (Eds.) (2003). Noun phrase structure in the languages of Europe. Berlín: Walter de Gruyter.

Rijkhoff, JAN (2002). The noun phrase. Nueva York: Oxford University Press.

SeILER, HANSJAKOB (1983). Possession as an operational dimension of language. Tubinga: Gunter Narr Verlag.

Smith-Stark, Thomas (enero, 2004). Property concepts in San Baltasar Chichicapan Zapotec. Ponencia presentada en The Annual Meeting of the Society for the Study of the Indigenous Languages of the Americas (SSILA). Boston, Massachusetts. 
Stewart, Donald (1966). Gramática del mazahua (Correcciones y comentarios de Doris Bartholomew). México: Instituto Lingüístico de Verano.

Vargas Bernal, Gloria (2013). Alineamiento e intransitividad escindida en mazahua (Tesis de maestría). Centro de Investigación y Estudios Superiores en Antropología Social, México.

Villard, StéPhanie (2013). Los conceptos de propiedad en el chatino oriental de Zacatepec. Proceedings of the Conference on Indigenous Languages of Latin America VI. Austin: Universidad de Texas. Recuperado de https:/www.ailla.utexas.org/sites/default/files/documents/ Villard_CILLA_VI.pdf 


\section{Anexo}

\section{Abreviaturas}

$\begin{array}{llll}1 & \text { primera persona } & \text { LXC } & \text { léxico } \\ 2 & \text { segunda persona } & \text { MED } & \text { mediano } \\ 3 & \text { tercera persona } & \text { NAR } & \text { narración } \\ \text { ART } & \text { artículo } & \text { NEG } & \text { negación } \\ \text { AUM } & \text { aumentativo } & \text { NP } & \text { nombre propio } \\ \text { COM } & \text { comitativo } & \text { NUM } & \text { numeral } \\ \text { CUANT } & \text { cuantificador } & \text { OBJ } & \text { objeto } \\ \text { DAT } & \text { dativo } & \text { ORD } & \text { orden } \\ \text { DEF } & \text { definido } & \text { PART } & \text { partitivo } \\ \text { DEL } & \text { delimitativo } & \text { PL } & \text { plural } \\ \text { DEM } & \text { demostrativo } & \text { POS } & \text { posesivo } \\ \text { DIAG } & \text { diálogo } & \text { PRED } & \text { predicativo } \\ \text { DIM } & \text { diminutivo } & \text { PRON } & \text { pronombre } \\ \text { DIME } & \text { dimensión } & \text { PRS } & \text { presente } \\ \text { DL } & \text { dual } & \text { PST } & \text { pasado } \\ \text { ENF } & \text { enfático } & \text { PSTR } & \text { pasado reciente } \\ \text { ELCD } & \text { elicitación directa } & \text { PTL } & \text { puntual } \\ \text { EST } & \text { estativo } & \text { REL } & \text { relativizador } \\ \text { EXCL } & \text { exclusivo } & \text { TAN } & \text { tono } \\ \text { FUT } & \text { futuro } & \text { SBJ } & \text { sujeto } \\ \text { INCL } & \text { inclusivo } & \text { SG } & \text { singular } \\ \text { IND } & \text { indefinido } & \text { SUST } & \text { sustantivo } \\ \text { LOC } & \text { locativo } & \text { TAempo adverbial } \\ & & & \end{array}$

